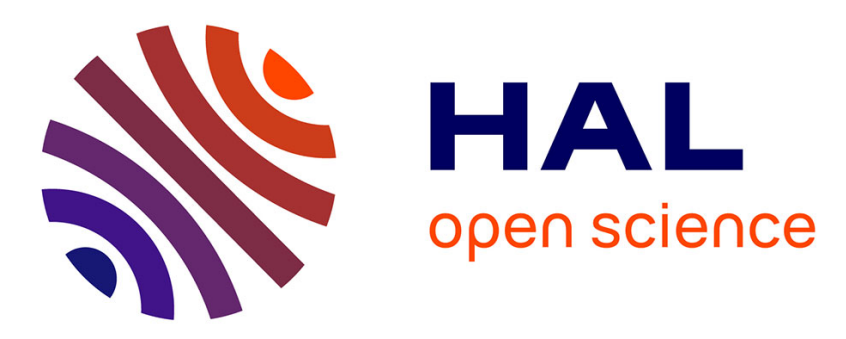

\title{
Après le bac professionnel ou technologique: la poursuite d'études jusqu'à bac +2 et sa rentabilité salariale en début de vie active
}

Madame Stéphanie Moullet

\section{- To cite this version:}

Madame Stéphanie Moullet. Après le bac professionnel ou technologique : la poursuite d'études jusqu'à bac +2 et sa rentabilité salariale en début de vie active. Economie et Statistique / Economics and Statistics, 2005, 388-389, pp.15 - 36. 10.3406/estat.2005.7168 . hal-03498089

\section{HAL Id: hal-03498089 \\ https://hal.science/hal-03498089}

Submitted on 20 Dec 2021

HAL is a multi-disciplinary open access archive for the deposit and dissemination of scientific research documents, whether they are published or not. The documents may come from teaching and research institutions in France or abroad, or from public or private research centers.
L'archive ouverte pluridisciplinaire HAL, est destinée au dépôt et à la diffusion de documents scientifiques de niveau recherche, publiés ou non, émanant des établissements d'enseignement et de recherche français ou étrangers, des laboratoires publics ou privés. 


\section{Après le bac professionnel ou technologique : la poursuite d'études jusqu'à bac +2 et sa rentabilité salariale en début de} vie active

\section{Madame Stéphanie Moullet}

\section{Citer ce document / Cite this document :}

Moullet Stéphanie. Après le bac professionnel ou technologique : la poursuite d'études jusqu'à bac +2 et sa rentabilité salariale en début de vie active. In: Economie et statistique, $n^{\circ} 388-389,2005$. Bilan Formation-Emploi (II) : Formation et qualité des emplois occupés. pp. 15-36;

doi : 10.3406/estat.2005.7168

http://www.persee.fr/doc/estat_0336-1454_2005_num_388_1_7168

Document généré le 23/02/2017 


\section{Résumé}

Le rendement salarial que les bacheliers professionnels et technologiques peuvent attendre de la poursuite de leurs études dépend de leur réussite, incertaine, au diplôme de niveau bac +2 . Le calcul de ce rendement est fondé sur le supplément de salaire que pourraient escompter ces bacheliers, compte tenu de leurs caractéristiques individuelles, s'ils poursuivaient leurs études en cas de réussite au niveau du diplôme bac +2 , ou sans obtenir de titre plus élevé. En tenant exclusivement compte de l'incertitude liée au risque d'échec au diplôme et au risque de chômage, les bacheliers professionnels qui poursuivent leurs études peuvent espérer après trois ans de vie active un supplément moyen de salaire de 5,6\% relativement à ceux qui arrêtent leur formation initiale au bac, de $+10,6 \%$ pour les bacheliers technologiques. Néanmoins, pour ces derniers, les caractéristiques des deux groupes (arrêt des études ou poursuite avec succès) diffèrent, et une simulation de la poursuite d'études pour les simples bacheliers ne leur attribue qu'un gain de 3,2 $\%$ en cas de succès au diplôme de bac +2 . Le rendement simulé de la poursuite d'études diplômantes par les bacheliers sortant immédiatement après leur réussite au bac professionnel est du même ordre de grandeur que celui obtenu par ceux qui ont effectivement poursuivi et réussi au niveau bac +2 (autour de $13 \%$ ). L'avantage retiré de la poursuite d'études tiendrait alors essentiellement à une meilleure rémunération des caractéristiques individuelles analogues à celles de ceux qui ont effectivement poursuivi et réussi au niveau bac +2 . Les bacheliers professionnels qui n'ont pas poursuivi au-delà auraient pu avoir intérêt à le faire, et ce même dans le cas d'un cursus non sanctionné par un diplôme (le rendement serait alors de 5,2 \%). Ces résultats reposent néanmoins sur l'hypothèse que les informations disponibles suffisent pour caractériser l'homogénéité ou la dissemblance des populations étudiées.

\section{Zusammenfassung}

Zweijähriges Studium nach dem Berufs-oder Technologieabitur und dessen lohnmaBiger Vorteil zu Beginn des Erwerbslebens

Welchen lohnmaBigen Nutzen die Absolventen eines Fachabiturs aus einen'l anschlieBenden zweijahrigen Studium ziehen, hangt vom Bestehen des Diploms ab. Die Berechnung dieses Nutzens basiert auf dem zusatzlichen Lohn, den diese Abiturienten aufgrund ihrer individuellen Merkmale erwarten konnten, wenn sie nach Bestehen dieses Diploms weiter studieren oder kein hoheres Diplom erwerben. Unter ausschlieBlicher Berücksichtigung der Unsicherheit im Hinblick auf das Nichtbestehen des Diploms oder die Arbeitslosigkeit konnen die Inhaber eines Berufsabiturs, die ihre Ausbildung fortsetzen, nach drei Jahren Erwerbstatigkeit mit einem durchschnittlichen Lohnzuwachs von 5,6\% gegenüber denen, die ihre Erstausbildung mit dem Abitur abschlieBen, und die Inhaber eines Technologieabiturs von 10,6\% rechnen. Bei Letzteren unterscheiden sich jedoch die Merkmale der beiden Gruppen (Beendigung der Ausbildung oder erfolgreiches Studium), sodass bei einer Simulation des Studiums die Inhaber lediglich eines Abiturs nur einen Zugewinn von 3,2\% im Falle des Bestehens des Diploms nach einem zweijahrigen Studium erlangen. Der simulierte Nutzen des Erwerbs eines Studienabschlusses für die Abiturienten, die unmittelbar nach Abschluss des Berufsgymnasiums ins Erwerbsleben eintreten, ist dagegen gleich groB wie bei denjenigen, die ein zweijahriges Studium absolvierten und erfolgreich abschlossen (etwa 13\%). Demnach bestünde der Vorteil eines Studiums im Wesentlichen in einer besseren Vergütung der individuellen Merkmale, die denen derjenigen entsprechen, die ein zweijahriges Studium absolvierten und erfolgreieh abschlossen. Die Inhaber eines Berufsabiturs, die danach nicht weiter studierten, hatten aber gut getan, dies zu tun, auch ohne Erwerb eines Diploms (der Nutzen lage bei 5,2\%). Diese Ergebnisse basieren allerdings auf der Hypothese, dassdieverfügbarenInformationenzurCharakterisierung der Homogenitat oder Unterschiedlichkeit der untersuchten Populationen ausreichen.

\section{Resumen}

Tras el bachillerato profesional o tecnológico: la continuaci6n de estudios durante dos anos y su rentabilidad salarial al iniciar la vida activa 
El rendimiento salarial que pueden alcanzar los bachilleres profesionales y tecnolégicos como consecuencia de sus estudios depende de la obtencién, incierta, de la titulacién de nivel bachiller +2 . El calculo de dicho rendimiento se basa en el suplemento salarial con el que podrfan contar estos bachilleres, conforme a sus caracterfsticas particulares, en casa de continuar sus estudios tras la obtencién de la titulacién de nivel bachiller $+2,0$ sin la obtencién dei grade mas elevado. Teniendo como (mica incertidumbre el. riesgo de no obtener la titulacién y, por ende, un empleo, los bachilleres profesionales que continuan sus estudios pueden esperar, tras tres anos de vida activa, un promedia de 5,6 \% mas en su salario que el de aquéllos que suspenden su formacién inicial de bachillerato, mas de 10,6 \% para los bachilleres tecnolégicos. No obstante, para estos ultimos, las caracterfsticas de los dos grupos (suspensién de estudios 0 continuacién con éxito) difieren, siendo asf que la continuacién de los estudios sélo atribuye un incremento de 3,2 \% a los simples bachilleres en casa de obtener la titulacién de nivel bachiller +2 . Por el contrario, el rendimiento proyectado de la continuacién de estudios sancionados con bachilleres egresados, después de haber obtenido inmediatamente el bachillerato profesional, es de una orden de magnitud similar a la conseguida por aquéllos que han continuado y obtenido el nivel bachiller +2 (alrededor de $13 \%$ ). La ventaja en la continuacién de estudios conllevarfa principalmente una mejor remuneracién de las caracteristicas individuales analogas a las de aquéllos que han continuado y obtenido el nivel bachiller +2 . Los bachilleres profesionales que no han proseguido con otros estudios hubieran podido estar interesados en hacerlo, yeso mismo en el casa de estudios universitarios en los que no se concede titulacién (el rendimiento serfa asf de 5,2 \%). No obstante, dichos resultados se basan en la hipétesis de que basta con las informaciones disponibles para caracterizar la homogeneidad 0 la diferencia de las poblaciones estudiadas.

\section{Abstract \\ After the Professional or Technological Baccalauréat: Wage Return at the Start of Working Life for Holders of a 2-Year Higher Education Diploma (Bac + 2)}

The wage return professional or technological baccalauréat holders can expect depends on whether or not they obtain a 2-year higher education diploma $(\mathrm{Bac}+2)$. This return is calculated according to the wage supplement they can expect to receive (taking into account individual characteristics) if they continue their studies beyond the 2-year higher education diploma, or if no further qualification is obtained. Allowing for uncertainty, stemming from the risk of diploma failure and unemployment, after three years of working life professional baccalauréat holders who continue their studies can hope to obtain an average salary supplement of $5.6 \%$ compared with those who stopped their initial training at baccalauréat level, a figure that rises to $10.6 \%$ for technological baccalauréat holders. However, when the differing characteristics of these two groups (Le. those who stop studying and those who continue their studies with success) are taken into account, baccalauréat holders obtaining a 2-year higher education diploma can expect a wage supplement of just $3.2 \%$. Studies have shown, however, that there is no difference between the wage return to professional baccalauréat holders pursuing further qualifications and the wage return to those who have actually studied for and obtained the 2-year higher education diploma (around 13\%). Those who continue their studies would be rewarded with better pay in recognition of individual characteristics which are similar to those characteristics of holders of a 2-year higher education diploma. Professional baccalauréat holders who have not continued to this level would have been weil advised to have done so, since even for courses not leading to a qualification the wage return would be $5.2 \%$. These results, however, depend upon the assumption that there is sufficient information available to characterise the similarities and differences of the populations studied. 


\title{
Après le bac professionnel ou technologique : la poursuite d'études jusqu'à bac +2 et sa rentabilité salariale en début de vie active
}

\author{
Stéphanie Moullet*
}

Le rendement salarial que les bacheliers professionnels et technologiques peuvent attendre de la poursuite de leurs études dépend de leur réussite, incertaine, au diplôme de niveau bac +2 . Le calcul de ce rendement est fondé sur le supplément de salaire que pourraient escompter ces bacheliers, compte tenu de leurs caractéristiques individuelles, s'ils poursuivaient leurs études en cas de réussite au niveau du diplôme bac +2 , ou sans obtenir de titre plus élevé.

En tenant exclusivement compte de l'incertitude liée au risque d'échec au diplôme et au risque de chômage, les bacheliers professionnels qui poursuivent leurs études peuvent espérer après trois ans de vie active un supplément moyen de salaire de 5,6\% relativement à ceux qui arrêtent leur formation initiale au bac, de $+10,6 \%$ pour les bacheliers technologiques.

Néanmoins, pour ces derniers, les caractéristiques des deux groupes (arrêt des études ou poursuite avec succès) diffèrent, et de ce fait une simulation de la poursuite d'études pour les simples bacheliers ne leur attribue qu'un gain de 3,2\% en cas de succès au diplôme de bac +2 .

Le rendement simulé de la poursuite d'études diplômantes par les bacheliers sortant immédiatement après leur réussite au bac professionnel est, en revanche, du même ordre de grandeur que celui obtenu par ceux qui ont effectivement poursuivi et réussi au niveau bac +2 (autour de $13 \%$ ). L'avantage retiré de la poursuite d'études tiendrait alors essentiellement à une meilleure rémunération des caractéristiques individuelles analogues à celles de ceux qui ont effectivement poursuivi et réussi au niveau bac +2 . Les bacheliers professionnels qui n'ont pas poursuivi au-delà auraient pu avoir intérêt à le faire, et ce même dans le cas d'un cursus non sanctionné par un diplôme (le rendement serait alors de $5,2 \%)$.

Ces résultats reposent néanmoins sur l'hypothèse que les informations disponibles suffisent pour caractériser l'homogénéité ou la dissemblance des populations étudiées.

\footnotetext{
* Stéphanie Moullet appartient au Céreq (Département des Entrées dans la Vie Active). Courriel : moullet@cereq.fr. L'auteur remercie Arnaud Dupray pour ses critiques et suggestions, ainsi que les deux rapporteurs anonymes de la revue.
} 
D epuis le début des années 1980, les effectifs de jeunes qui entrent dans l'enseignement supérieur se sont accrus. La quasi-totalité des bacheliers généraux y accèdent, la part des bacheliers issus de séries autres que générales croissant. Plus de quatre bacheliers technologiques sur cinq poursuivent leurs études dès la rentrée suivante en s'orientant le plus souvent vers des filières courtes, en particulier en STS, qui scolarisent plus de la moitié d'entre eux. La différence majeure entre les bacheliers généraux et technologiques s'observe dans le type d'orientation. Ainsi, en 1996, les premiers sont près de $58 \%$ à opter pour la voie des études longues à l'université alors que les seconds ne sont que deux sur dix à s'y inscrire, $68 \%$ s'orientant vers un IUT ou en STS (Lemaire, 2004). Plus récemment, seule la proportion de bacheliers professionnels poursuivant leurs études continue d'augmenter. Alors même que le bac professionnel a pour vocation première de préparer à l'entrée immédiate sur le marché du travail, une proportion non négligeable de détenteurs de ce diplôme (18 \%) (1) choisissent d'entrer dans l'enseignement supérieur, essentiellement dans des filières professionnelles. En 1998, près d'un tiers des jeunes arrête les études une ou deux années après le bac, qu'un diplôme de niveau bac +2 (BTS, DUT ou Deug) ait été obtenu ou non. On compte 90000 jeunes qui ont entamé des études supérieures mais sans obtenir de diplôme supérieur au bac (Giret et al., 2003) et ce sont les jeunes titulaires d'un bac professionnel ou technologique qui, plus souvent que les bacheliers généraux, sont dans cette situation d'échec. Les bacheliers technologiques représentent en effet, en 1998, $60 \%$ des sorties sans diplôme de filières professionnelles contre près de $30 \%$ des sorties diplômées d'un DUT et environ six diplômés de BTS sur dix. Quant aux bacheliers professionnels, ils constituent $16 \%$ des non-diplômés issus de filières professionnelles et dans le même temps, moins de $3 \%$ de la population des diplômés (Thomas, 2003).

Pour la population des bacheliers tant technologiques que professionnels, la question de la poursuite dans l'enseignement supérieur comme alternative à l'entrée sur le marché du travail se pose donc d'autant plus que le risque d'échec au diplôme bac +2 est important. Compte tenu de la finalité différente de ces deux types de bac - l'entrée dans la vie active pour le bac professionnel et la poursuite d'études dans l'enseignement supérieur professionnel pour le bac technologique -, et des taux de poursuite divergents qui en découlent, ces deux populations constituent deux cas de figures pertinents à partir desquels il est possible de conduire une analyse comparative. Ces constats fondent notre double objectif.

Le premier est de déterminer comment les caractéristiques des titulaires d'un bac technologique ou professionnel contribuent à leur décision d'entrée dans l'enseignement supérieur, à l'obtention du diplôme de niveau bac +2 , et à l'accès à l'emploi. En particulier, on tentera d'isoler l'influence des parcours scolaires antérieurs.

Le second est d'évaluer la rentabilité salariale d'investissements éducatifs correspondant à trois types de sortie du système éducatif : immédiatement après le bac, après avoir poursuivi dans l'enseignement supérieur sans obtenir de diplôme, et avec un diplôme de niveau bac +2 .

\section{Le bac professionnel ou technologique et après : caractéristiques des populations étudiées}

L'importance prise par l'objectif de mener $80 \%$ d'une génération au niveau du bac a fait de ce diplôme un repère prédominant en matière d'éducation (Hanchane et Verdier, 2003). Sept jeunes sur dix des générations récentes ont atteint le niveau du bac, $65 \%$ des jeunes sont bacheliers, dont $26 \%$ des bacheliers technologiques et $11 \%$ des bacheliers professionnels. De facto, le bac est devenu un carrefour important des parcours scolaires où les questions de poursuite d'études et d'orientation se posent de la façon la plus décisive (2). Le bac technologique ouvre logiquement sur les filières courtes de l'enseignement supérieur conduisant à un BTS ou un DUT (Dubois et Raulin, 1997). Quant aux bacheliers professionnels, plus des deux tiers de ceux qui poursuivent leurs études s'orientent vers les formations professionnelles qui débouchent sur un BTS.

Parmi les détenteurs d'un bac technologique qui ont poursuivi leurs études au-delà de ce diplôme, 35 \% quittent pourtant en 1998 le système éducatif sans diplôme supplémentaire. C'est aussi le cas de six bacheliers professionnels sur dix qui ont poursuivi dans des forma-

1. Ce taux de poursuite passe à $23,9 \%$ (Lemaire, 1998) si l'on tient compte du fait que certains bacheliers professionnels, un sur trois, poursuit la formation dans l'enseignement supérieur par la voie de l'alternance avec un contrat d'apprentissage ou de qualification.

2. Des travaux antérieurs (Epiphane et Hallier, 1996) à partir de données portant sur les bacheliers généraux et technologiques de 1998 se sont intéressés à leur devenir scolaire 
tions post-bac. Ces bacheliers, professionnels et technologiques, alimentent de ce fait l'essentiel $\mathrm{du}$ flux des jeunes qui quittent l'enseignement supérieur sans diplôme.

De l'obtention du baccalauréat jusqu'à l'emploi occupé trois ans après la sortie de formation initiale, les cheminements se distinguent selon le type de bac. Sur la base de données d'insertion de la génération des jeunes sortis de formation initiale en 1998 (l'enquête Génération 98 du Céreq, cf. encadré 1), on traite de manière distincte les jeunes diplômés de bac professionnel et de bac technologique sortis de formation initiale, du niveau
IV (diplômé du bac) au niveau III (diplômé du niveau bac +2$)(3)$.

Pour les bacheliers professionnels, l'arbre d'orientation (cf. schéma 1) est basé sur une population de 5031 individus dont 20,7 \% poursuivent leur formation initiale jusqu'au niveau bac +2 , seulement un tiers (347) obtenant leur diplôme. Les taux d'emploi après trois ans de

3. Pour ces deux types de bac, la sortie de formation à un niveau supérieur au bac +2 est peu fréquente: seulement $6 \%$ des bacheliers professionnels et $14 \%$ des bacheliers technologiques qui sont entrés dans l'enseignement supérieur sortent de formation initiale en 1998 au-delà du niveau III.

Schéma 1

L'arbre d'orientation pour les bacheliers professionnels

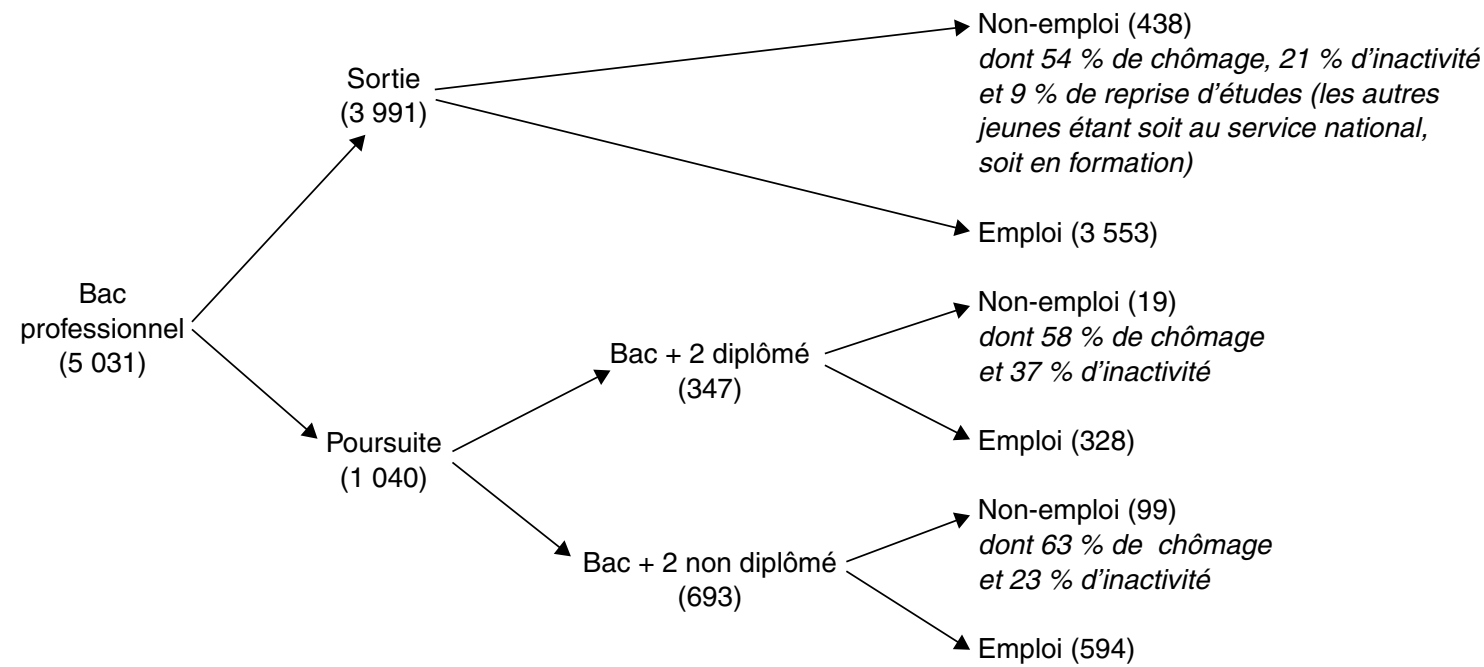

Source : Génération 98, Céreq.

Encadré 1

\section{LES DONNÉES DE GÉNÉRATION 98}

Les données sont issues de l'enquête Génération 98 réalisée par le Centre d'Études et de Recherches sur les Qualifications (Céreq) au printemps 2001. Elles concernent 55000 sortants du système éducatif en 1998 tous niveaux de formation et toutes spécialités de formation confondus parmi les 750000 primo-sortants en 1998

L'enquête a pour objet de rendre compte des différentes composantes des parcours d'insertion professionnelle : elle fournit des informations individuelles, sociodémographiques et relatives au parcours scolaire ainsi que des informations relatives aux différents emplois occupés.

Les données, en partie rétrospectives, permettent ainsi d'analyser les trois premières années de vie active au regard du cursus de formation initiale réalisé. Elles autorisent donc la comparaison des modalités d'insertion professionnelle des jeunes selon leur niveau d'éducation dans une même conjoncture économique. Cette source permet de saisir les différences de situation, notamment salariale, à la date de l'enquête. 
vie active est de $89 \%$ pour l'ensemble des 5031 bacheliers professionnels de l'échantillon, de $85,7 \%$ dans le cas de sortie sans diplôme de niveau bac +2 et de 94,5 \% lorsque le diplôme est obtenu. Le faible taux de poursuite est cohérent avec la spécificité du bac professionnel qui prépare ses détenteurs à une insertion directe sur le marché du travail. Toutefois, pour ceux entrant dans l'enseignement supérieur, la probabilité empirique de succès au diplôme est de $33 \%$.

Pourlesbachelierstechnologiques(cf.schéma 2), la population étudiée est de 7563 individus dont $82,1 \%$ poursuivent la formation initiale jusqu'au niveau bac +2 , où près de $54 \%$ d'entre eux (3 371) obtiennent leur diplôme. La configuration est opposée à la précédente puisque seule une minorité (1 352 sur 7 563) de titulaires d'un baccalauréat technologique sortent à ce niveau. Comparativement aux bacheliers professionnels qui poursuivent, le taux de réussite au diplôme est de 21 points supérieur (54\% contre $33 \%$ ), suggérant que la différence entre les caractéristiques (les attributs) des diplômés et celles des jeunes en échec soit plus importante à l'issue de la filière professionnelle du bac que de celle de la filière technologique.

Le taux d'emploi après trois ans de vie active est de $76,8 \%$ pour les bacheliers technologiques sortant au niveau du bac, de $82,3 \%$ dans le cas de sortie sans diplôme de niveau bac +2 et de $92,1 \%$ lorsque le diplôme est obtenu. Le taux d'accès à l'emploi est d'autant plus faible que le niveau de sortie est bas. Pour autant, ceux qui ne sont pas en emploi peuvent être chômeurs ou inactifs, la probabilité de reprise d'études étant particulièrement élevée pour ce public (Thomas, 2003).

\section{Des caractéristiques individuelles différentes...}

Les populations de jeunes qui poursuivent des études supérieures de niveau bac +2 et ceux qui quittent l'école à l'issue de l'enseignement secondaire ont connu des scolarités distinctes selon le type de bac obtenu (cf. tableaux A et B en annexe 1).

Près de $32 \%$ sont en retard en classe de sixième parmi les sortants au niveau d'un bac professionnel, ils sont $34 \%$ dans ce cas parmi les non-diplômés de niveau bac +2 et moins de $28 \%$ pour les diplômés de ce niveau. Le retard scolaire rapproche également les jeunes sortants immédiatement après le bac technologique de ceux qui poursuivent au-delà du bac sans obtenir de diplôme supérieur (avec respectivement $20 \%$ et $19 \%$ contre $13 \%$ pour les diplômés de niveau III).

La classe de troisième est une section générale dans plus de 9 cas sur 10 quel que soit le type de sortie de formation initiale pour les détenteurs d'un bac technologique. Près de $77 \%$ des sortants au bac professionnel ont suivi une troisième générale contre $80 \%$ pour ceux qui sortent sans un diplôme bac +2 et $88 \%$ pour ceux qui ont obtenu le diplôme. La nature de la classe après la troisième distingue aussi les différentes populations: les sortants au niveau

Schéma 2

L'arbre d'orientation pour les bacheliers technologiques

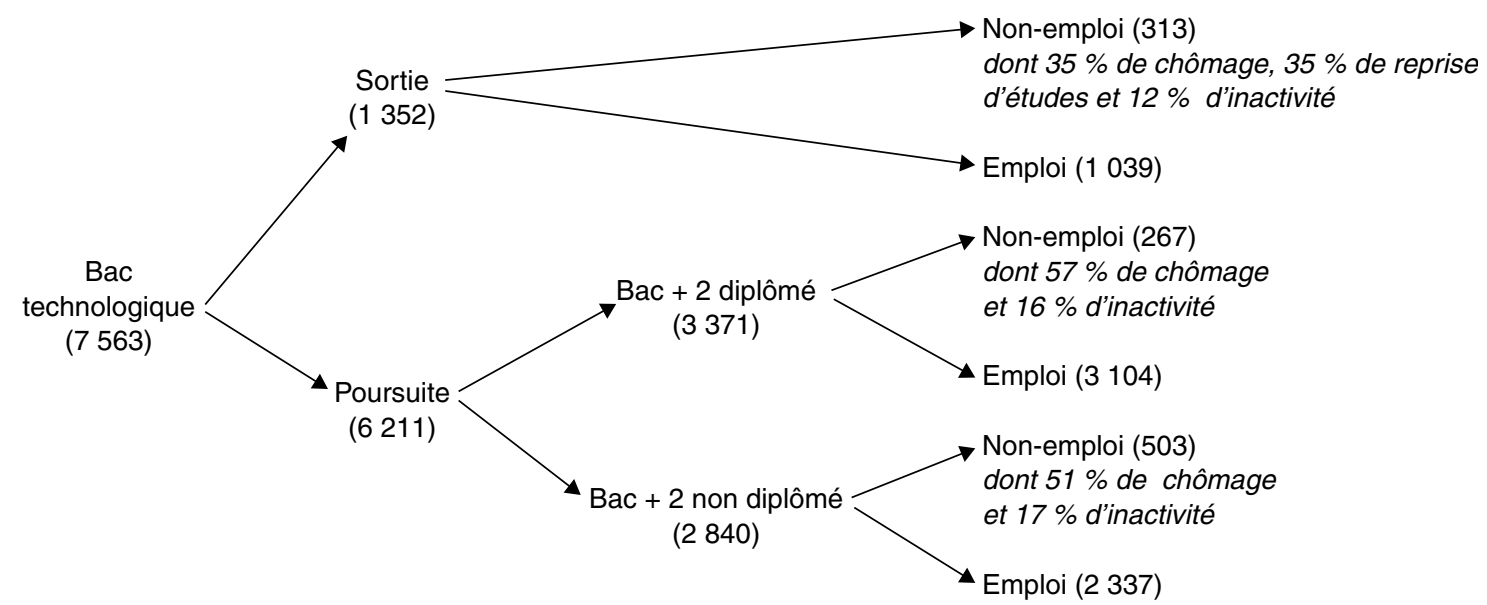

Source : Génération 98, Céreq. 
du bac professionnel sont $79 \%$ à posséder un CAP ou BEP (quelle qu'en soit la voie, scolaire ou apprentissage), cette proportion étant de $76 \%$ pour les non-diplômés de l'enseignement supérieur, soit sensiblement plus que celle des bacs $+2(69 \%)$. Les bacheliers technologiques ont moins souvent fréquenté une seconde générale quand ils quittent l'école à l'issue du bac $(42 \%)$ que s'ils poursuivent au-delà $(55 \%)$; $38 \%$ des premiers sont passés par un CAP ou $\mathrm{BEP}$, proportion plus de deux fois supérieure à celle des seconds.

Les bacheliers professionnels qui arrêtent leurs études à ce stade ont pour plus de la moitié (51\%) un diplôme de spécialité industrielle, alors que ceux qui poursuivent ont plus souvent une spécialité tertiaire (pour $68 \%$ d'entre eux). La spécialité tertiaire est en revanche davantage la caractéristique de ceux qui s'arrêtent au bac technologique (avec $73 \%$ ) que de ceux qui poursuivent la formation initiale $(61 \%$ ont un bac tertiaire, 70 \% s'ils sont non-diplômés de bac +2 ).

Quand ils sont diplômés du supérieur, les bacheliers professionnels ont un BTS, de spécialité tertiaire dans $62 \%$ des cas. S'ils quittent le système éducatif sans diplôme après le bac, ils sont issus de Deug pour $43 \%$ d'entre eux, et pour $34 \%$ d'une filière professionnelle tertiaire (c'est-à-dire une formation débouchant sur un BTS ou un DUT de spécialité tertiaire). $14 \%$ des bacheliers technologiques diplômés du supérieur et sortant au niveau bac +2 détiennent un DUT, près de $83 \%$ ont un BTS (pour plus de la moitié industriel). Pour les non-diplômés, près de 6 sur 10 ont échoué en STS ou IUT. Enfin, l'échec à l'issue d'une filière professionnelle est tout aussi important parmi les détenteurs d'un bac professionnel que pour ceux, dans la même situation, qui possèdent un bac technologique $(57 \%$ et $58 \%)$.
Conformément à leur concentration dans les filières industrielles, les hommes quittent le système éducatif plus tôt que les femmes lorsqu'ils obtiennent un bac professionnel : $60 \%$ des sortants au niveau du bac sont des hommes contre $49 \%$ parmi les jeunes qui poursuivent leurs études au-delà du bac. Le cas inverse s'observe chez les bacheliers technologiques avec respectivement $37 \%$ et $55 \%$ d'hommes. Quel que soit leur bac d'origine, ceux qui obtiennent un diplôme supérieur de niveau bac +2 sont plus souvent des hommes que ce n'est le cas chez les jeunes en échec à ce niveau.

La catégorie sociale de la mère à la fin des études est la même quel que soit le niveau de sortie du système éducatif et le type de bac obtenu. En revanche, avoir un père cadre est une caractéristique plus fréquente chez les jeunes diplômés de bac +2 que pour les sortants au niveau du bac ( $7,5 \%$ contre $15 \%$ de père cadre chez les bac professionnels, $10,5 \%$ contre $16 \%$ chez les bac technologiques). Enfin, l'origine étrangère du père ou de la mère est davantage propre aux jeunes qui poursuivent au-delà du bac plutôt qu'à ceux qui arrêtent leur formation initiale à ce stade. Elle semble cependant être associée à une moindre réussite au diplôme supérieur, en particulier dans le cas des bacheliers professionnels.

\section{... et des conditions d'entrée dans la vie active différentes}

Les taux d'emploi à trois ans des jeunes poursuivant leurs études au-delà du bac sont plus élevés, que cette poursuite soit ou non sanctionnée par l'obtention d'un diplôme pour les bacheliers technologiques, et seulement lorsque les études supérieures aboutissent à un diplôme pour les bacheliers professionnels (cf. tableau 1). Le gain salarial lié à la poursuite des études n'est

Tableau 1

L'insertion professionnelle des bacheliers professionnels et technologiques selon le niveau de fin d'études

\begin{tabular}{|l|c|c|c|c|c|c|}
\hline $\begin{array}{c}\text { Niveau de sortie de } \\
\text { formation initiale }\end{array}$ & \multicolumn{2}{|c|}{$\begin{array}{c}\text { Répartition des sortants } \\
\text { (en \%) }\end{array}$} & \multicolumn{2}{c|}{$\begin{array}{c}\text { Proportion de ceux qui ont un } \\
\text { emploi (en \%) }\end{array}$} & \multicolumn{2}{c|}{$\begin{array}{c}\text { Salaires mensuels moyens } \\
\text { (en euros) }\end{array}$} \\
\hline & $\begin{array}{c}\text { Bacheliers } \\
\text { professionnels } \\
\text { (total }: 100)\end{array}$ & $\begin{array}{c}\text { Bacheliers } \\
\text { technologiques } \\
\text { (total }: 100)\end{array}$ & $\begin{array}{c}\text { Bacheliers } \\
\text { professionnels }\end{array}$ & $\begin{array}{c}\text { Bacheliers } \\
\text { technologiques }\end{array}$ & $\begin{array}{c}\text { Bacheliers } \\
\text { professionnels }\end{array}$ & $\begin{array}{c}\text { Bacheliers } \\
\text { technologiques }\end{array}$ \\
\hline Bac & 79,3 & 17,9 & 89,0 & 76,8 & 1020,6 & 946,0 \\
\hline Bac + 2, sans diplôme & 13,8 & 37,6 & 85,7 & 82,3 & 1038,7 & 1042,6 \\
\hline Bac + 2, avec diplôme & 6,9 & 44,5 & 94,5 & 92,1 & 1229,2 & 1195,7 \\
\hline
\end{tabular}

Lecture : parmi les détenteurs d'un bac professionnel sortis de formation initiale en 1998 immédiatement après le bac ou au niveau bac +2 , 6,9 \% ont le diplôme bac +2 . Parmi ces derniers, 94,5 \% occupent un emploi à la date d'enquête et gagnent en moyenne 1 229,2 euros mensuel, primes incluse, en mars 2001.

Source : Génération 981 ère interrogation, Céreq. 
important que lorsque le diplôme de bac +2 est obtenu.

L'insertion professionnelle des diplômés de $\mathrm{bac}+2$ tout comme celle des sortants de niveau IV supérieur dépend largement de la filière de formation suivie voire également $d u$ type de bac pour les non-diplômés. À ce niveau, les filières professionnelles continuent $d$ 'assurer les conditions d'insertion les meilleures (Giret et al., 2003) et l'obtention du diplôme reste déterminante pour l'accès à l'emploi. En particulier, après trois ans de vie active, les sortants de STS sans diplôme, détenteurs d'un bac professionnel ou technologique, ont des taux de chômage de $8 \%$, soit près de deux fois plus que ceux des diplômés. À l'issue d'un premier cycle universitaire, le taux de chômage passe de $8 \%$ pour les diplômés à $12 \%$ et $14 \%$ pour les non-titulaires respectivement bacheliers professionnels et technologiques. De ce point de vue, leur situation est nettement moins favorable que celle des bacheliers professionnels et technologiques qui n'ont pas poursuivi leurs études au-delà (respectivement $7 \%$ et $9 \%$ ) (Thomas, 2003).

En termes de caractéristiques des emplois détenus après trois ans de vie active, les différences sont également notables en fonction du bac et du niveau de sortie de formation initiale.

Si en 2001, $45 \%$ des bacheliers professionnels occupent un emploi d'ouvrier, ils ne sont que $22 \%$ dans ce cas parmi ceux qui ont poursuivi leurs études sans obtenir de diplôme de niveau bac +2 et $9 \%$ parmi ceux qui l'ont obtenu (cf. tableaux D et $\mathrm{E}$ en annexe 1). La part des emplois de cadres ou professions intermédiaires pour les bacheliers professionnels passe de $21 \%$ pour les simples bacheliers à $56 \%$ pour les titulaires d'un bac +2 . De la même façon, le poids des professions intermédiaires et des cadres fait plus que doubler entre les sortants au niveau du bac technologique et les diplômés de bac $+2(27 \%$ à $59 \%)$.

Dans 94 \% des situations, les jeunes diplômés $\mathrm{du}$ supérieur travaillent à temps plein, près de dix points de plus que pour les sortants au niveau bac.

Enfin, en termes de contrat de travail, près de 7 titulaires d'un bac professionnel sur 10 ont un CDI ou sont fonctionnaires, plus que parmi les jeunes poursuivant sans diplôme (6 sur 10) mais moins que les diplômés bac $+2(77 \%)$. Ce constat est différent pour les détenteurs d'un bac technologique : l'accès à l'emploi à durée indé- terminée concerne moins les sortants au niveau bac $(51 \%)$ que ceux issus du supérieur sans diplôme $(57 \%)$, moins encore que les diplômés bac $+2(74 \%)$.

Les bacheliers professionnels qui ont poursuivi leurs études sans obtenir de diplôme supplémentaire occupent plus souvent des emplois aidés que les jeunes sortis au niveau du bac (16\% contre $10 \%)$. En revanche, la part des emplois aidés, trois ans après la fin des études, est la même pour les bacheliers technologiques que pour les non-diplômés de niveau bac +2 , titulaires de ce bac $(18 \%)$.

$\mathrm{Au}$ total, en termes de caractéristiques individuelles comme en termes de conditions d'insertion professionnelle, c'est l'obtention du diplôme de bac +2 davantage que la poursuite d'études en soi qui différencie ces populations. De plus, les éléments du parcours scolaire sont des caractéristiques distinctives des bacheliers selon qu'ils poursuivent leurs études et qu'ils quittent la formation initiale diplômés ou non. Dans ce qui suit, le caractère déterminant de ces parcours de formation initiale dans la décision de poursuite d'études et l'obtention du diplôme va être analysé.

Dans la mesure où la population des jeunes diplômés de bac +2 en emploi en 2001, tout comme celle des jeunes salariés sortis immédiatement après le bac, ne résultent pas de sélections aléatoires au sein de la population des sortants de niveau IV supérieur et III, apprécier l'avantage salarial résultant de la poursuite d'études dans le supérieur va nécessiter le recours à une méthodologie prenant en compte ces sélections.

\section{L'influence du parcours scolaire dans l'enseignement secondaire : de la poursuite d'études à l'accès à l'emploi}

L'effet des variables traduisant le parcours scolaire est analysé en tenant compte de l'influence de l'origine sociale (catégorie sociale du père et de la mère) et du pays de naissance des parents sur la poursuite d'études au-delà du bac, la réussite au diplôme de niveau bac +2 et l'accès à l'emploi. L'importance de l'origine sociale sur l'orientation et le niveau d'études espéré est connue (Coëffic, 1998, Fabre et Moullet, 2004, Goux et Maurin, 1997, Hanchane et Verdier, 2003), mais d'autres déterminants comme ceux liés à la construction des itinéraires scolaires ont peu fait l'objet d'investigations, à l'exception de celles de Dupray et Recotillet (2005). 
Le rôle central des facteurs tels que la catégorie sociale des parents est depuis longtemps démontré pour expliquer le niveau d'éducation des enfants. À l'étape charnière du parcours éducatif que constitue le bac, les variables susceptibles de traduire les opportunités de financement de la formation supérieure et les capacités (aptitudes) individuelles manifestent tous leurs effets.

Les schémas 1 et 2 distinguent les situations sur le marché du travail (en emploi vs. en nonemploi) selon le niveau de sortie de formation. Sachant que les individus qui quittent le système éducatif au niveau du bac, par exemple, ont de caractéristiques spécifiques les distinguant de l'ensemble des jeunes bacheliers et dans la mesure où des facteurs explicatifs - observés ou non - de leur décision de mettre fin à leurs études peuvent également expliquer leur situation vis-à-vis de l'emploi, on tient compte de l'interdépendance des situations (de niveau de formation et d'emploi) à l'aide d'un modèle qualitatif trivarié de forme Probit (cf. encadré 2).

Les résultats de l'estimation du modèle Probit trivarié figurent dans le tableau 2. Pour les bacheliers professionnels et technologiques, seuls les enfants de père cadre ont une probabilité de poursuite d'études au niveau bac +2 plus élevée que les autres. De plus, cette caractéristique du père continue de jouer un rôle favorable sur les chances de succès au diplôme supérieur après un bac professionnel ou technologique. Avoir une mère ouvrière augmente la probabilité de sortie du système éducatif immédiatement après le bac professionnel. C'est le cas aussi pour ces bacheliers dont les mères sont artisans ou commerçants. L'origine étrangère des parents constitue un facteur allant dans le sens de la poursuite des études dans l'enseignement supérieur, en cohérence avec les résultats de Caille et Vallet (1995), mais en revanche plutôt défavorable à la réussite au diplôme de niveau bac +2 pour les bacheliers technologiques.

En ce qui concerne la probabilité d'emploi à trois ans, pour les bacheliers professionnels, avoir une mère ouvrière ou artisan-commerçantchef d'entreprise plutôt qu'employée l'améliore. En revanche, avoir un père cadre plutôt qu'employé réduit leur chance d'être en emploi. Ce résultat apparemment étonnant s'explique par le fait que les enfants de cadre surtout s'ils sortent sans diplôme du supérieur sont les plus prompts à reprendre des études, prenant acte sans doute plus aisément que des jeunes de milieux socio- culturels plus défavorisés de leur déficit de formation initiale (Eckert, 2002).

Pour les bacheliers technologiques, la catégorie socioprofessionnelle du père n'a aucun effet sur l'accès à l'emploi alors qu'une mère en nonemploi à la fin des études réduit les chances d'emploi à trois ans. Si cette dernière est artisan-commerçant-chef d'entreprise, la probabilité d'emploi s'en trouve améliorée.

Toutes choses égales par ailleurs, un homme a une plus grande propension à poursuivre des études au-delà du bac, quel qu'il soit. Tant le risque d'échec au diplôme bac +2 que celui de non-emploi sont également réduits dans ce cas pour les bacheliers technologiques. En revanche, de ces points de vue, hommes et femmes ne se différencient pas au sein de la population des bacheliers professionnels.

Le retard scolaire à l'entrée en sixième que l'on peut considérer comme une approximation de l'«aptitude» de l'élève, est supposé être un frein à la poursuite d'études et diminuer les chances de succès dans l'obtention du diplôme de niveau $b a c+2$. Les résultats montrent que son effet n'est pas significatif pour expliquer l'arrêt de la formation initiale après l'obtention du bac professionnel ou technologique, mais il joue à l'encontre de la réussite au diplôme pour les jeunes qui poursuivent leurs études au-delà du bac, quel qu'il soit.

Le type de classe de troisième (générale ou technologique, d'insertion ou Segpa (4) n'a pas d'effet sur la probabilité de poursuite d'études dans le supérieur pour les bacheliers technologiques. Être passé par une classe de troisième autre que générale réduit cependant les chances de poursuite d'études pour les bacheliers professionnels et réduit la probabilité d'obtention du diplôme de niveau bac +2 pour les bacheliers technologiques et professionnels.

Si la classe suivie après la troisième correspond à une formation professionnelle plutôt que générale ou technologique, la probabilité d'arrêt des études au niveau du bac technologique s'en trouve fortement accrue, et ce d'autant plus s'il s'agit d'une formation par apprentissage (BEP, CAP). Le même effet de la formation professionnelle par apprentissage après la classe de troisième s'observe pour les bache-

4. Segpa: Section d'enseignement général et professionnel adapté. 
Tableau 2

Probabilité d'occuper un emploi après trois ans de vie active étant données les probabilités de poursuite d'études après le bac et de réussite au diplôme de niveau bac +2 (modèles Probit trivariés)

\begin{tabular}{|c|c|c|c|c|}
\hline & \multicolumn{2}{|c|}{ Bacheliers professionnels } & \multicolumn{2}{|c|}{ Bacheliers technologiques } \\
\hline & Coefficient & $P$-value & Coefficient & $P$-value \\
\hline \multicolumn{5}{|c|}{ Les déterminants de la probabilité d'emploi } \\
\hline $\begin{array}{l}\text { Constante } \\
\text { Sortie de formation initiale au niveau du bac } \\
\text { Échec au diplôme de niveau bac }+2 \\
\text { Homme } \\
\text { Absence d'emploi pendant les études } \\
\text { Existence d'emploi de vacances }\end{array}$ & $\begin{array}{r}2,6326 \\
-0,8726 \\
-1,3165 \\
0,0574 \\
-0,1649 \\
0,0738\end{array}$ & $\begin{array}{l}0,000 \\
0,000 \\
0,000 \\
0,188 \\
0,000 \\
0,007\end{array}$ & $\begin{array}{r}1,2726 \\
-0,5166 \\
-0,4791 \\
0,2181 \\
-0,1546 \\
0,1921\end{array}$ & $\begin{array}{l}0,000 \\
0,000 \\
0,000 \\
0,000 \\
0,002 \\
0,000\end{array}$ \\
\hline $\begin{array}{l}\text { Spécialité industrielle du bac } \\
\text { (Référence : bac tertiaire) }\end{array}$ & 0,3227 & $0,000^{*}$ & 0,0165 & $0,710^{*}$ \\
\hline $\begin{array}{l}\text { Catégorie sociale de la mère à la fin des étud } \\
\text { (Référence : employé) } \\
\text { Artisan, commerçant } \\
\text { Cadre } \\
\text { Technicien } \\
\text { Ouvrier } \\
\text { Inactive, chômeur }\end{array}$ & $\begin{array}{r}0,2940 \\
-0,0365 \\
0,0017 \\
0,1451 \\
-0,0371\end{array}$ & $\begin{array}{l}0,000 \\
0,698 \\
0,987 \\
0,025 \\
0,437\end{array}$ & $\begin{array}{r}0,1368 \\
-0,0522 \\
0,0531 \\
0,0092 \\
-0,1182\end{array}$ & $\begin{array}{l}0,071 \\
0,409 \\
0,539 \\
0,868 \\
0,012\end{array}$ \\
\hline $\begin{array}{l}\text { Catégorie sociale du père à la fin des études } \\
\text { (Référence : employé) } \\
\text { Artisan, commerçant } \\
\text { Cadre } \\
\text { Ouvrier } \\
\text { Technicien } \\
\text { Inactive, chômeur }\end{array}$ & $\begin{array}{r}0,0105 \\
-0,2060 \\
0,0079 \\
0,0314 \\
-0,0149\end{array}$ & $\begin{array}{l}0,862 \\
0,004 \\
0,914 \\
0,532 \\
0,829\end{array}$ & $\begin{array}{r}-0,0140 \\
-0,0361 \\
0,0137 \\
0,0680 \\
-0,0645\end{array}$ & $\begin{array}{l}0,810 \\
0,530 \\
0,810 \\
0,164 \\
0,319\end{array}$ \\
\hline $\begin{array}{l}\text { Classe après la troisième } \\
\text { (Référence : seconde générale) } \\
\text { Seconde technologique } \\
\text { BEP, CAP apprentissage } \\
\text { BEP, CAP voie scolaire } \\
\text { Autres secondes }\end{array}$ & $\begin{array}{r}-0,1768 \\
0,5039 \\
-0,0374 \\
0,2211\end{array}$ & $\begin{array}{l}0,052 \\
0,000 \\
0,513 \\
0,032\end{array}$ & $\begin{array}{l}0,0836 \\
0,2095 \\
0,1971 \\
0,1259\end{array}$ & $\begin{array}{l}0,062 \\
0,176 \\
0,000 \\
0,552\end{array}$ \\
\hline \multicolumn{5}{|c|}{ Les déterminants de la probabilité d'échec au diplôme de niveau bac +2} \\
\hline $\begin{array}{l}\text { Constante } \\
\text { Homme } \\
\text { Retard scolaire à l'entrée en troisième } \\
\text { Père français ou né en France } \\
\text { Mère française ou née en France }\end{array}$ & $\begin{array}{r}1,3790 \\
0,0688 \\
0,1121 \\
-0,0483 \\
-0,1783\end{array}$ & $\begin{array}{l}0,000 \\
0,171 \\
0,019 \\
0,625 \\
0,084\end{array}$ & $\begin{array}{r}0,4866 \\
-0,3234 \\
0,1178 \\
-0,1401 \\
-0,1057\end{array}$ & $\begin{array}{l}0,000 \\
0,000 \\
0,005 \\
0,035 \\
0,126\end{array}$ \\
\hline $\begin{array}{l}\text { Catégorie sociale de la mère à la fin des étud } \\
\text { (Référence : employé) } \\
\text { Artisan, commerçant } \\
\text { Cadre } \\
\text { Technicien } \\
\text { Ouvrier } \\
\text { Inactive, chômeur }\end{array}$ & $\begin{array}{r}0,3278 \\
0,1870 \\
-0,0122 \\
0,0022 \\
0,0528 \\
\end{array}$ & $\begin{array}{l}0,001 \\
0,132 \\
0,927 \\
0,979 \\
0,434\end{array}$ & $\begin{array}{r}-0,0852 \\
-0,0463 \\
0,0030 \\
-0,0406 \\
-0,0039\end{array}$ & $\begin{array}{l}0,169 \\
0,443 \\
0,966 \\
0,431 \\
0,927\end{array}$ \\
\hline $\begin{array}{l}\text { Catégorie sociale du père à la fin des études } \\
\text { (Référence : employé) } \\
\text { Artisan, commerçant } \\
\text { Cadre } \\
\text { Ouvrier } \\
\text { Technicien } \\
\text { Inactif, chômeur }\end{array}$ & $\begin{array}{r}-0,0755 \\
-0,2778 \\
0,0245 \\
0,0658 \\
0,2050\end{array}$ & $\begin{array}{l}0,333 \\
0,002 \\
0,800 \\
0,345 \\
0,047\end{array}$ & $\begin{array}{r}-0,0705 \\
-0,0965 \\
-0,0727 \\
0,0443 \\
0,1322\end{array}$ & $\begin{array}{l}0,150 \\
0,049 \\
0,170 \\
0,310 \\
0,023\end{array}$ \\
\hline $\begin{array}{l}\text { Classe après la troisième } \\
\text { (Référence : seconde générale) } \\
\text { Seconde technologique } \\
\text { BEP, CAP apprentissage } \\
\text { BEP, CAP voie scolaire } \\
\text { Autres secondes }\end{array}$ & $\begin{array}{r}-0,0922 \\
0,7053 \\
0,1864 \\
0,6320\end{array}$ & $\begin{array}{l}0,409 \\
0,000 \\
0,008 \\
0,000\end{array}$ & $\begin{array}{r}-0,1461 \\
0,5486 \\
0,3627 \\
0,2463\end{array}$ & $\begin{array}{l}0,000 \\
0,000 \\
0,000 \\
0,191\end{array}$ \\
\hline $\begin{array}{l}\text { Autres troisièmes } \\
\text { (Référence : troisième générale) }\end{array}$ & 0,1265 & 0,033 & 0,1380 & 0,042 \\
\hline \multicolumn{5}{|c|}{ Les déterminants de la probabilité de sortie de formation initiale au niveau du bac } \\
\hline $\begin{array}{l}\text { Constante } \\
\text { Homme } \\
\text { Retard scolaire à l'entrée en sixième } \\
\text { Père français ou né en France } \\
\text { Mère française ou née en France }\end{array}$ & $\begin{array}{r}0,4454 \\
-0,0906 \\
-0,0209 \\
0,1079 \\
0,1178\end{array}$ & $\begin{array}{l}0,000 \\
0,049 \\
0,514 \\
0,048 \\
0,037\end{array}$ & $\begin{array}{r}-1,1746 \\
-0,3607 \\
0,0591 \\
0,1704 \\
0,1639\end{array}$ & $\begin{array}{l}0,000 \\
0,000 \\
0,200 \\
0,009 \\
0,016\end{array}$ \\
\hline
\end{tabular}


liers professionnels. En revanche, pour ces derniers, le passage par une seconde technologique plutôt que générale accroît les chances de poursuivre des études dans l'enseignement supérieur. Avoir suivi une seconde technologique relativement à une seconde générale est un facteur favorable à l'obtention du diplôme de niveau bac +2 , au contraire du passage par un BEP pour les bacheliers technologiques. Pour les titulaires d'un bac professionnel, l'obtention du diplôme est particulièrement freinée pour ceux passés par une première année de BEP-CAP par apprentissage et dans une moindre mesure, par voie scolaire. Un déficit de connaissances générales pourrait être un facteur défavorable à la réussite dans l'enseignement supérieur.
Le passage par un BEP ou CAP continue d'avoir un effet sur la probabilité d'occuper un emploi trois ans après la fin des études. Pour les jeunes en possession d'un bac professionnel, détenir un emploi à cette date est d'autant plus probable, toutes choses égales par ailleurs, qu'ils sont passés par un BEP ou CAP par apprentissage alors que le passage par une classe de seconde technologique est pénalisant. À niveau de sortie de formation initiale donné, les bacheliers technologiques sont relativement favorisés dans l'accès à l'emploi dans le cas d'un passage par un BEP-CAP par voie scolaire.

Pour l'accès à l'emploi, la spécialité du bac technologique (industriel ou tertiaire) ne distingue pas les sortants bacheliers. Pour les titu-

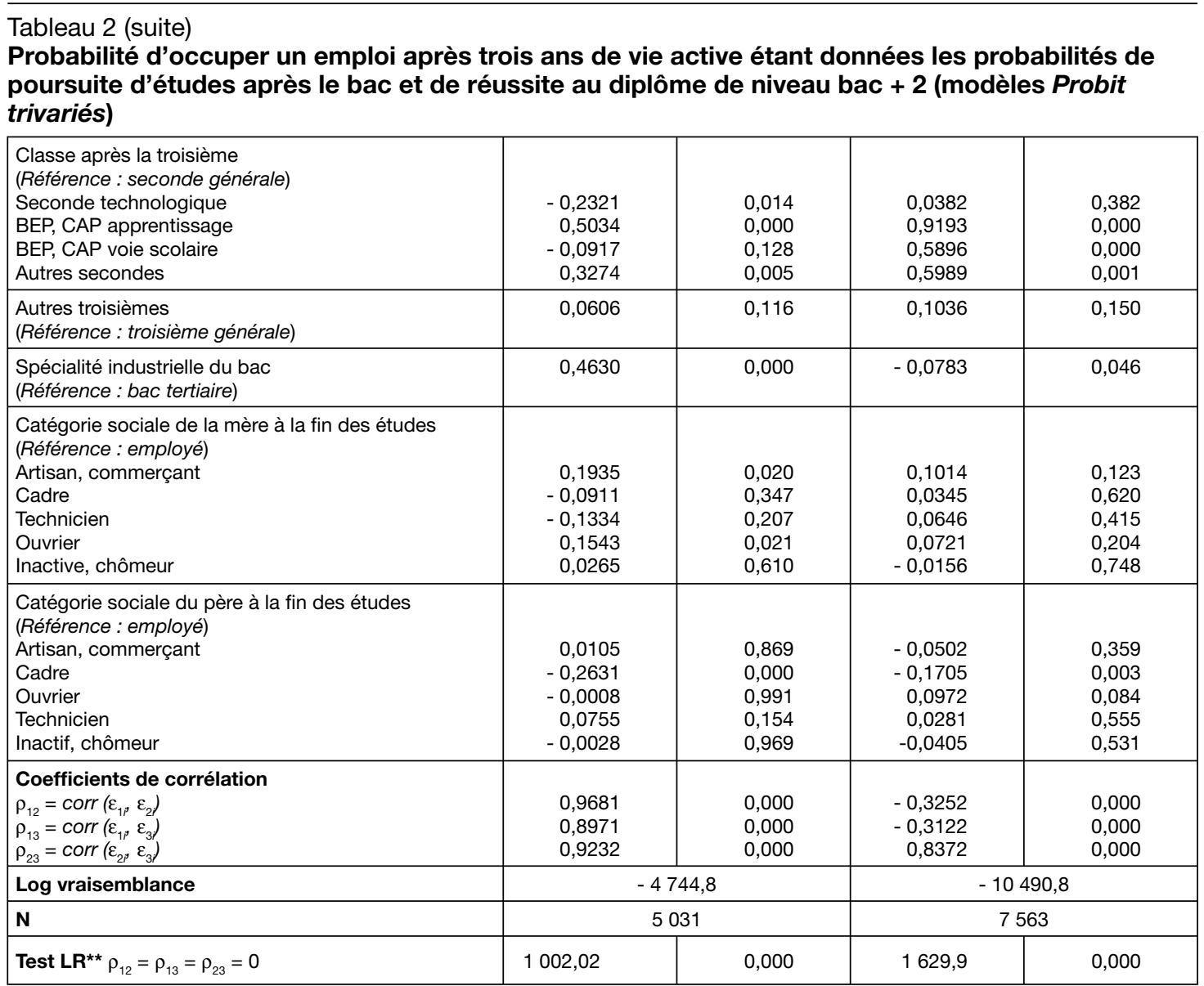

Lecture : $\left(^{*}\right)$ les p-values indiquent le seuil de significativité du paramètre estimé. La spécialité industrielle du bac professionnel augmente significativement au seuil de $1 \%$ les chances d'emploi à trois ans par rapport aux spécialités tertiaires. Pour les bacheliers technologiques, spécialités industrielles et tertiaires ne se distinguent pas pour expliquer la probabilité d'emploi de manière significative : le risque d'erreur à conclure à un effet de la spécialité dépasse $70 \%$.

(*) le test du ratio de vraisemblance permet de rejeter l'hypothèse d'absence de corrélation entre les variables inobservées des trois équations. Pour la population des détenteurs d'un bac professionnel, les variables inobservées déterminant la probabilité de ne pas poursuivre d'études post-bac, celles déterminant la probabilité d'échec au diplôme de bac +2 et celles explicatives de la probabilité d'occuper un emploi, après trois ans de vie active, sont corrélées positivement entre elles. Pour les détenteurs d'un bac technologique, les facteurs inobservés déterminant la probabilité de quitter la formation initiale immédiatement après le bac et les facteurs non observés explicatif de l'échec au diplôme de bac +2 sont les uns comme les autres négativement corrélés les variables inobservées explicatives de l'accès à l'emploi. Enfin, les facteurs non observés de la probabilité d'échec au diplôme de bac + 2 et ceux déterminant l'accès à l'emploi sont corrélés positivement.

Source : Génération 98, 1 ère interrogation, Céreq. 
Encadré 2

\section{MODÈLE PROBIT TRIVARIÉ ET ENDOGÉNÉITÉ DE LA POURSUITE D’ÉTUDES}

Afin de contrôler l'endogénéité de la poursuite d'études, un système de variables qualitatives dépendantes décrivant le type de sortie de formation initiale (immédiatement après le bac, au niveau IV supérieur) ainsi que l'accès à l'emploi à la date d'enquête est estimé par des modèles de forme Probit. Les niveaux de fin d'études retenus interviennent comme variables explicatives de la situation d'emploi. Formellement, les trois processus en cause, reliés entre eux, sont modélisés à partir de variables latentes $\mathrm{y}_{\mathrm{si}}^{*}, s=1,2$ ou 3 , de la façon suivante:

$$
\begin{aligned}
& y_{1 \mathrm{i}}=\left\{\begin{array}{lll}
1 & \text { si } & y_{1 \mathrm{i}}^{*}>0 \\
0 & \text { si } & y_{1 \mathrm{i}}^{*} \leq 0
\end{array} \text { où } \quad y_{1 \mathrm{i}}^{*}=x_{1 \mathrm{i}}{ }^{\prime} \beta_{1}+\varepsilon_{1 \mathrm{i}}\right. \\
& y_{2 i}=\left\{\begin{array}{lll}
1 & \text { si } & y_{2 i}^{*}>0 \\
0 & \text { si } & y_{2 i}^{*} \leq 0
\end{array} \text { où } \quad y_{2 i}^{*}=x_{2 i}{ }^{\prime} \beta_{2}+\varepsilon_{2 i}\right. \\
& y_{3 i}=\left\{\begin{array}{lll}
1 & \text { si } & y_{3 i}^{*}>0 \\
0 & \text { si } & y_{3 i}^{*} \leq 0
\end{array} \quad \text { où } \quad y_{3 i}^{*}=x_{3 i}{ }^{\prime} \beta_{3}+\gamma_{1} y_{1 i}+\gamma_{2} y_{2 i}+\varepsilon_{3 i}\right.
\end{aligned}
$$

La variable dichotomique $y_{1}$ correspond à la sortie de formation initiale immédiatement après l'obtention du bac, $y_{2}$ à la sortie de formation initiale au niveau de formation IV supérieur. Enfin, $y_{3}$ prend la valeur 1 si l'individu est en emploi à la date d'enquête, 0 sinon. Une spécification de type Probit repose sur l'hypothèse de normalité de la loi des composantes d'erreur des modèles de régression sous-jacents, la normalisation à 1 des variances étant nécessaire pour des raisons d'identification. On a :

$\left(\begin{array}{l}\varepsilon_{1} \\ \varepsilon_{2} \\ \varepsilon_{3}\end{array}\right) \rightarrow \mathrm{N}(0, \Sigma)$ et $\Sigma=\left(\begin{array}{ccc}1 & \rho_{12} & \rho_{13} \\ \rho_{12} & 1 & \rho_{23} \\ \rho_{13} & \rho_{23} & 1\end{array}\right)$

À moins que les termes d'erreurs soient indépendants, des conditions d'exclusion de variables doivent nécessairement être imposées pour que les paramètres $\gamma$ soient identifiés. La contribution individuelle à la fonction de vraisemblance s'écrit comme ci-dessous :

$L=\prod_{i=1}^{n} P\left(y_{1}=1, y_{2}=1, y_{3}=1\right) P\left(y_{1}=0, y_{2}=1, y_{3}=1\right) p\left(y_{1}=0, y_{2}=0, y_{3}=1\right) P\left(y_{1}=1, y_{2}=1, y_{3}=0\right)$

$P\left(y_{1}=0, y_{2}=1, y_{3}=0\right) P\left(y_{1}=0, y_{2}=0, y_{3}=0\right)$

avec

$P\left(y_{1}=1, y_{2}=1, y_{3}=1\right)=\int_{-x_{1} \beta_{1}-x_{2} \beta_{2}-x_{3} \beta_{3}-\gamma_{1}-\gamma_{2}}^{+\infty} \int_{3}^{+\infty} \int_{1}^{+\infty}\left(0,0,0, \rho_{12}, \rho_{13}, \rho_{23}\right) d \varepsilon_{3} d \varepsilon_{2} d \varepsilon_{1}$

la probabilité que l'individu n'ait pas poursuivi d'études supérieures et soit en emploi à la date d'enquête ; $\phi_{3}$ est la fonction de densité de la loi normale trivariée.

$P\left(y_{1}=0, y_{2}=1, y_{3}=1\right)=\int_{-\infty}^{-x_{1} \beta_{1}} \int_{-x_{2} \beta_{2}}^{+\infty} \int_{-x_{3} \beta_{3}-\gamma_{2}}^{+\infty} \phi_{3}\left(0,0,0, \rho_{12}, \rho_{13}, \rho_{23}\right) d \varepsilon_{3} d \varepsilon_{2} d \varepsilon_{1}$

la probabilité que l'individu soit sorti de formation initiale au niveau IV supérieur (non-diplômés de bac +2 ) et soit en emploi à la date de l'enquête ;

$P\left(y_{1}=0, y_{2}=0, y_{3}=1\right)=\int_{-\infty}^{-x_{1} \beta_{1}-x_{2} \beta_{2}} \int_{-\infty}^{+\infty} \int_{-x_{3} \beta_{3}}^{+\infty} \phi_{3}\left(0,0,0, \rho_{12}, \rho_{13}, \rho_{23}\right) d \varepsilon_{3} d \varepsilon_{2} d \varepsilon_{1}$

la probabilité que l'individu soit sorti de formation initiale diplômé de bac +2 et soit en emploi à la date de l'enquête ;

$P\left(y_{1}=1, y_{2}=1, y_{3}=0\right)=\int_{-x_{1} \beta_{1}}^{+\infty} \int_{-x_{2} \beta_{2}}^{+\infty} \int_{-\infty}^{-x_{3} \beta_{3}-\gamma_{1}-\gamma_{2}} \phi_{3}\left(0,0,0, \rho_{12}, \rho_{13}, \rho_{23}\right) d \varepsilon_{3} d \varepsilon_{2} d \varepsilon_{1}$

la probabilité que l'individu n'ait pas poursuivi d'études supérieures et ne soit pas en emploi à la date de l'enquête ;

$P\left(y_{1}=0, y_{2}=1, y_{3}=0\right)=\int_{-\infty}^{-x_{1} \beta_{1}} \int_{-x_{2} \beta_{2}}^{+\infty} \int_{-\infty}^{-x_{3} \beta_{3}-\gamma_{2}} \phi_{3}\left(0,0,0, \rho_{12}, \rho_{13}, \rho_{23}\right) d \varepsilon_{3} d \varepsilon_{2} d \varepsilon_{1}$

la probabilité que l'individu soit sorti de formation initiale au niveau IV supérieur et ne soit pas en emploi à la date de l'enquête et 
laires d'un bac professionnel, les spécialités industrielles (et agricoles) favorisent cet accès relativement aux spécialités tertiaires. En différentiant encore les deux bacs, le niveau de sortie du système éducatif détermine fortement la probabilité d'emploi trois ans plus tard. Pour les bacheliers professionnels, sortir au niveau IV supérieur est un frein à l'emploi davantage encore que si l'entrée sur le marché du travail a lieu immédiatement à l'issue du bac. En revanche pour les bacheliers technologiques, sortir de formation initiale non diplômé de niveau bac +2 ou sortir au niveau IV de formation défavorise de manière comparable l'accès à l'emploi.

L'hypothèse d'absence de corrélation entre les variables individuelles inobservées explicatives d'une part, de la probabilité de sortir de formation initiale après le bac, d'autre part, du risque d'échec au diplôme bac +2 et de la probabilité d'emploi après trois ans de vie active, est rejetée par un test du ratio de vraisemblance (cf. tableau 2), ce qui est cohérent avec le choix de modélisation retenu. De plus, les corrélations entre les caractéristiques inobservées explicatives de l'accès à l'emploi et celles explicatives de l'échec au diplôme bac +2 , comme avec les déterminants inobservés de l'arrêt des études au bac sont significatives à la fois pour les bacheliers professionnels et pour les bacheliers technologiques.

$\mathrm{Au}$ total, certaines caractéristiques relatives au parcours scolaire en collège et lycée influencent la probabilité de poursuite d'études au niveau $\mathrm{bac}+2$ et la réussite à ce diplôme. Ces résultats confirment l'intérêt de la prise en compte de ces variables de trajectoires scolaires pour étudier les conditions de poursuite d'études post-bac et d'insertion professionnelle. En particulier, cette poursuite et le risque d'échec au diplôme qui lui est associé agissent non seulement sur la probabilité d'occuper un emploi à la date d'enquête mais aussi dans la détermination des salaires, dans la mesure où ces étapes sélectionnent des populations spécifiques. Les attributs propres à ceux qui poursuivent ou, tout aussi particuliers, aux diplômés de bac +2 impliquent qu'ignorer ce caractère singulier risque de conduire à des conclusions erronées sur l'efficacité salariale de la poursuite d'études, diplômante ou non. Traiter du rendement salarial de la formation suppose dès lors de contrôler ces effets de sélection puisqu'une part de la rémunération est attribuable à la valorisation des caractéristiques explicatives de ces sélections. Préalablement, il est possible d'évaluer les rendements espérés de la poursuite d'études après le bac au moyen de fonction de

Encadré 2 (suite)

$P\left(y_{1}=0, y_{2}=0, y_{3}=0\right)=\int_{-\infty}^{-x_{1} \beta_{1}-x_{2} \beta_{2}-x_{3} \beta_{3}} \int_{-\infty}^{\infty} \int_{-\infty}\left(0,0,0, \rho_{12}, \rho_{13}, \rho_{23}\right) d \varepsilon_{3} d \varepsilon_{2} d \varepsilon_{1}$

la probabilité que l'individu soit sorti de formation initiale diplômé de bac +2 et ne soit pas en emploi à la date de l'enquête.

En raison de l'intégration multidimensionnelle de la fonction de vraisemblance, on recourt pour son estimation aux techniques de simulation à l'aide de la procédure GHK (cf. Greene, 2003) et à l'extension à la programmation de Stata proposée par Terracol (2002) dans le cas de variables endogènes (ce qui est de cas ici des variables $y_{1}$ et $\left.y_{2}\right)$.

Variables explicatives et variables d'exclusion

Concernant les probabilités d'achever la formation initiale à l'issue du bac et celle de sortie sans diplôme dans le cas où la formation se poursuit dans l'enseignement supérieur, les facteurs explicatifs regroupent des variables informatives du parcours de formation initiale - l'existence d'un retard scolaire à l'entrée en classe de sixième, le type de classe de troisième suivi et celui de la classe suivie après l'année suivante. Le sexe, la catégorie sociale des parents en fin d'études et le pays de naissance de chacun d'eux sont également pris en compte.

Pour expliquer la probabilité d'être en emploi à la date d'enquête, on retient l'existence éventuelle d'emploi en cours d'études ou de vacances, la spécialité de formation du bac, la classe suivie après la troisième, le sexe ainsi que la CS des deux parents en 1998

Pour identifier l'ensemble des paramètres du modèle, l'estimation requiert que certaines explicatives des deux premières équations ne soient pas incluses dans la troisième. On choisit d'écarter la variable de retard scolaire à l'entrée du collège et le type de classe de troisième dans la mesure où le niveau de sortie est supposé intégrer l'ensemble de ces caractéristiques d'itinéraire scolaire et constitue une proxy des capacités individuelles pour accéder à l'emploi. 
gains classiques et des probabilités moyennes d'échec au diplôme bac +2 et d'emploi.

\section{Rendements espérés de la poursuite d'études post-bac}

Le rendement salarial espéré de la poursuite d'études supérieures (cf. tableau 3), qu'elle débouche sur un diplôme ou pas, est de 10,9 \% pour les détenteurs d'un bac professionnel et de $13,1 \%$ pour les bacheliers technologiques, en emploi à la date d'enquête. Ce rendement global est décomposé entre celui tiré de la poursuite non diplômante et celui permis par l'obtention d'un diplôme. Pour les bacheliers professionnels, le supplément salarial acquis avec le diplôme bac +2 est quatre fois plus important que le supplément que l'individu peut espérer à l'issue d'une poursuite d'études sans diplôme (21\% contre 5,3\%). L'apport du diplôme n'est que de deux fois et demi supérieur à celui escompté d'une poursuite non diplômante pour les bacheliers technologiques $(18,1 \%$ contre $7 \%)$.

La plus-value se joue essentiellement sur la réussite au diplôme $\mathrm{bac}+2$, et ceci est plus accentué pour les bacheliers professionnels que pour les bacheliers technologiques.

Ces rendements espérés sont soumis à une double incertitude. La première tient au risque d'échec au diplôme bac +2 , ce risque étant estimé en moyenne à $93 \%$ pour les bacheliers professionnels et à près de $55 \%$ pour les bacheliers technologiques. En tenant compte de ce risque, les rendements espérés deviennent alors plus faibles, particulièrement pour les bacheliers professionnels: la poursuite dans l'enseignement supérieur, parce qu'elle est assortie d'une probabilité d'échec au diplôme, n'accroîtrait en moyenne que de $6,4 \%$ les gains des bacheliers professionnels et de près de $12 \%$ les salaires des bacheliers technologiques. L'écart plus fort $(12 \%$ contre $6,4 \%$ au lieu de $13,1 \%$ contre $10,9 \%$ ) entre bacheliers constaté ici par rapport aux résultats sur les rendements globaux est lié à la probabilité d'échec au diplôme plus importante pour les bacheliers professionnels que pour les bacheliers technologiques. La seconde incertitude tient au risque de non-emploi, risque d'autant plus grand que le diplôme supérieur au bac n'est pas obtenu. Une manière d'en tenir compte consiste à calculer les rendements de la poursuite d'études en les pondérant non seulement par la probabilité empirique d'obtenir le diplôme mais aussi par la probabilité d'emploi moyenne selon que le diplôme est obtenu ou pas.

$\mathrm{Au}$ total donc, sachant que l'obtention du diplôme bac +2 est incertaine et que, de cette obtention, dépend pour partie la probabilité de non-emploi après trois de vie active (chômage ou inactivité pour l'essentiel), les bacheliers professionnels qui poursuivent leurs études peuvent espérer un supplément moyen de salaire de 5,6\% relativement à ceux qui arrêtent leur formation initiale au bac (cf. tableau 3). Ce surcroît de salaire espéré est de $10,6 \%$ pour les bacheliers technologiques (5). Sur la base de ce premier résultat, la poursuite d'études apparaîtrait comme deux fois plus avantageuse pour les

5. Parce qu'ils sont associés à des populations spécifiques, ces rendements sont difficilement comparables aux résultats d'autres travaux empiriques. Sur données françaises et sur des populations masculines âgées de 16 à 55 ans, Lollivier et Pollet (2003) établissent aux alentours de $10 \%$ le rendement d'une année d'études supplémentaire lorsque la formation initiale est traitée comme une variable endogène et que l'effet de l'éducation sur l'accès à l'emploi est intégré.

Tableau 3

Taux de rendements salariaux espérés de la poursuite d'études post-bac

\begin{tabular}{|c|c|c|}
\hline & Bacheliers professionnels & Bacheliers technologiques \\
\hline $\begin{array}{l}\text { Rendement espéré de la poursuite (1) } \\
\text { (Référence : bac) }\end{array}$ & & \\
\hline Poursuite non diplômante & 5,3 & 7,0 \\
\hline Poursuite diplômante & 21,0 & 18,1 \\
\hline $\begin{array}{l}\text { Rendement espéré avec prise en compte de l'incertitude sur } \\
\text { l'obtention du diplôme (2) }\end{array}$ & 6,4 & 12,0 \\
\hline $\begin{array}{l}\text { Rendement espéré avec prise en compte de l'incertitude sur } \\
\text { l'obtention du diplôme et la situation d'emploi (3) }\end{array}$ & 5,6 & 10,6 \\
\hline \multicolumn{3}{|c|}{$\begin{array}{l}\text { 1. Rendements issus de fonctions de gains incluant également le sexe, l'expérience professionnelle, l'ancienneté dans l'emploi et la } \\
\text { spécialité du bac. } \\
\text { 2. Rendement obtenu en calculant une moyenne des rendements associés à la sortie de formation initiale au niveau IV supérieur et } \\
\text { à la sortie au niveau III de formation, par rapport à une sortie au bac (issus de l'estimation de fonctions de gains) pondérée par les } \\
\text { probabilités empiriques de ces sorties. } \\
\text { 3. Rendement obtenu en calculant une moyenne des rendements associés à la sortie de formation initiale au niveau IV supérieur et } \\
\text { à la sortie au niveau III de formation pondérée par les probabilités empiriques d'échec au diplôme bac + } 2 \text { et les probabilités moyen- } \\
\text { nes d'emploi correspondantes. }\end{array}$} \\
\hline
\end{tabular}


bacheliers technologiques que pour les jeunes issus de bac professionnels.

\section{Salaires prédits et avantages comparatifs : quelle efficacité de la poursuite d'études et quelle prime à l'obtention du diplôme ?}

Améliorer l'évaluation des rendements de la poursuite d'études postérieures au baccalauréat conduit à prendre en compte les corrélations entre le salaire perçu par un individu et ses probabilités de poursuite, de réussite au diplôme et d'emploi, et aussi à considérer que ces rendements sont variables entre groupes d'individus (définis par le niveau de sortie de formation initiale).

Les résultats précédents montrent d'une part que les individus ont des profils qui diffèrent selon le type de sortie après le bac professionnel et le bac technologique, et d'autre part, que certaines de leurs caractéristiques individuelles sont des facteurs explicatifs de leur devenir scolaire postérieur au bac. Si de plus, les effets sur les salaires des caractéristiques individuelles sont propres à chaque type de sortie et de bacheliers - ce que l'estimation des différentes fonctions de gains permet de vérifier - alors on ne peut raisonner sur la base des écarts de salaires moyens entre les différentes populations de sortants pour évaluer le gain salarial additionnel moyen que procure une poursuite d'études selon qu'elle est diplômante ou qu'elle ne l'est pas : ce qu'auraient gagné en moyenne les sortants au bac professionnel s'ils étaient sortis diplômés de bac +2 diffère de ce que gagnent effectivement (en moyenne) les titulaires de diplôme de bac +2 , issus d'un bac professionnel. L'analyse en termes d'efficacité de la poursuite d'études post-bac nécessite un exercice de simulation de salaires moyens pour chaque population compte tenu des caractéristiques moyennes de chacune et compte tenu aussi de la rémunération salariale de ces attributs. On calcule donc, par exemple, le salaire moyen « potentiel » des bacheliers professionnels $s$ 'ils sortaient diplômés de bac +2 sur la base de deux éléments : leurs caractéristiques individuelles et les rémunérations que les employeurs attribuent à chacune de ces caractéristiques pour les diplômés $\mathrm{de} b \mathrm{bc}+2$. On intègre également ce qui relève des effets de sélection, autrement dit ce qui tient aux caractéristiques explicatives du type de sortie et de la situation d'emploi (et de leurs rétributions par les employeurs) (cf. encadré 3 ).
Les salaires moyens prédits et les rendements associés figurent dans le tableau 4 pour les bacheliers professionnels et le tableau 5 pour les bacheliers technologiques.

Les écarts de salaires moyens des titulaires d'un bac professionnel entre niveaux de sortie montrent que le gain tient essentiellement à l'obtention du diplôme (cf. tableau 4). En effet, l'écart salarial entre les diplômés de bac +2 et les jeunes sortis immédiatement après l'obtention du bac est plus de onze fois plus grand (écart de 208,6 euros mensuels, soit 1229,2-1020,6) que le supplément de gains entre les jeunes sortis dès leur réussite au bac et les jeunes qui ont poursuivi au-delà sans diplôme (écart de 18,1 euros mensuels, soit 1038,7-1020,6).

Ces écarts traduisant aussi des différences de caractéristiques individuelles entre les trois populations de sortants, ils donnent une idée imparfaite du véritable supplément de revenu auquel pourraient s'attendre les sortants bacheliers s'ils poursuivaient leurs études, qu'ils échouent ou réussissent par la suite.

Les résultats obtenus sur la base des salaires simulés établissent pour les sortants immédiatement après leur réussite au bac professionnel un rendement de la poursuite d'études diplômante $(12,9 \%)$ peu différent de celui des bacheliers professionnels effectivement sortis de formation initiale avec un diplôme supérieur $(13,6 \%)$ (cf. tableau 4-B). Pour les sortants au niveau IV supérieur $(i=2)$, le rendement espéré d'une poursuite diplômante par rapport à la situation où ils auraient quitté l'école immédiatement après le bac est inférieur à celui des sortants bacheliers $(8,5 \%$ contre $12,9 \%)$. Il semble que leurs caractéristiques individuelles soient plus pénalisantes que celles des bacheliers sortis dès leur réussite au bac ou avec un diplôme de $\mathrm{bac}+2$

Pour les jeunes passés par un bac professionnel, la prime à la poursuite d'études relève en priorité de l'obtention du diplôme. En d'autres termes, pour deux sous-populations sur trois, le bénéfice marginal issu du diplôme (par rapport à une poursuite sans diplôme) est supérieur à celui tiré d'une poursuite non diplômante par rapport à une sortie immédiatement après l'obtention du bac.

Lorsqu'on identifie au sein de l'écart de salaire moyen entre deux types de sortie de formation initiale ce qui relève d'un effet de valorisation 
(différence de paiement à caractéristiques données) et ce qui tient à un effet de caractéristiques (différence de caractéristiques à paiement identique), il apparaît (cf. tableau 4-C) que pour la population des détenteurs d'un bac professionnel, les gains escomptés résultant de la poursuite d'études ou de l'obtention du diplôme tiennent pour l'essentiel à l'effet de valorisation. Bien plus, les poursuivants postbac qui échouent au diplôme de bac +2 possèdent des caractéristiques moins favorables que celles des sortants juste après leur réussite au bac. En revanche, lorsque l'on compare les jeunes sortis immédiatement après le bac professionnel et les diplômés de bac $+2,63 \%$ de la différence de salaire est dû à des rétributions différentes que font les employeurs pour des caractéristiques individuelles identiques. De ce fait, $37 \%$ de l'écart du revenu moyen observé $(76,5 / 208,6$ soit 37 \%) des diplômés de bac +2 passés par un bac professionnel résultent des caractéristiques singulières de ces individus relativement à celles de l'ensemble des titulaires d'un tel bac.

\section{Encadré 3 \\ SALAIRES SIMULÉS ET DÉCOMPOSITION DES ÉCARTS DE GAINS ENTRE TYPES DE SORTIE DE FORMATION INITIALE}

En matière de choix éducatifs, ce que l'on observe pour l'ensemble des individus, autrement dit, dans cette étude, les observations par type de sortie, ne peut être considéré comme résultant de tirages aléatoires dans la population totale. Le modèle Probit trivarié constitue alors la première étape d'une procédure d'estimation en deux temps. La seconde étape consiste à corriger des différentes sélections (celles liée à la détention d'un emploi à la date d'enquête et celles liées au niveau de formation initiale). Pour ce faire, on introduit dans les équations de salaire correspondant à chaque type de sortie (cf. annexe 2) les différentes probabilités estimées de sélection. Ces trois probabilités (d'emploi, d'échec au diplôme, de sortie après le bac), calculées à partir des prédicteurs de l'estimation du modèle Probit trivarié, permettent de contrôler les biais de sélection (Wooldrigde, 2001). Dans cette seconde étape, les écarts-types sont corrigés de l'hétéroscédasticité (White, 1980). Si les questions de biais de sélection sont depuis longtemps jugées centrales dans le domaine des décisions d'éducation (Willis et Rosen, 1979), il reste que la méthode de correction en deux étapes même la plus standard (Heckman, 1979) soulève encore un ensemble de critiques (Puhani, 2000). Par ailleurs, si la modélisation retenue ici pour les processus de poursuite d'études et d'emploi nous semble la plus appropriée, on n'ignore pas que les valeurs prédites à partir des estimations de la seconde étape sont sensibles au type de modélisation adopté en première étape (Hilmer, 2001).

Pour les équations de salaire, outre donc les trois probabilités estimées du type de sortie et d'emploi, on retient des variables explicatives dites de capital humain : le sexe, l'âge en 1998, la spécialité du bac, le temps de travail, l'ancienneté en emploi et l'expérience professionnelle. Une autre estimation a été réalisée en tenant alors compte des principales caractéristiques de l'emploi occupé à la date d'enquête. On a retenu pour ce faire la PCS occupée, le type de contrat de travail, le secteur d'activité et la taille de l'entreprise. Les résultats obtenus sont de même nature que ceux exposés dans les tableaux 4 et 5 .

Les paramètres estimés des équations de salaires permettent de calculer les salaires prédits pour chaque type de sortie du système éducatif, pour les valeurs moyennes des caractéristiques individuelles.

Soit $j=1,2$ ou 3 le type de sortie de formation initiale et $i=1,2$ ou 3 l'indice de l'individu moyen. Pour chaque $i$, on calcule les gains prédits de la façon suivante:

$E\left(W_{i j} / Z, y_{1}, y_{2}, y_{3}\right)=E\left(W_{i j}\right)=\hat{\alpha}_{j}{ }^{\prime} \bar{Z}_{i}+\hat{\theta}_{P_{1} j} \bar{P}_{1 i}+\hat{\theta}_{P_{2} j} \bar{P}_{2 i}$

$+\hat{\theta}_{\mathrm{P}_{3} j} \overline{\mathrm{P}}_{3 \mathrm{i}}$ pour $j=1,2,3$.

avec $y_{1}$ la variable de sortie de formation immédiatement après l'obtention du bac, $y_{2}$ la variable d'échec au diplôme de niveau bac +2 et $y_{3}$ celle d'emploi.

Chaque ensemble des coefficients estimés $\left(\hat{\alpha}_{j}, \hat{\theta}_{P_{1} j}, \hat{\theta}_{P_{2}}, \hat{\theta}_{P_{3} j}\right)$ est celui de l'équation de salaire estimée pour chaque type de sortie $j$ (trois par type de bac).

En s'inspirant des travaux précurseurs de Blinder (1973) et Oaxaca (1973), l'écart de rémunération entre deux types de sortie peut se décomposer comme suit, par exemple pour les sortants bac +2 diplômés $(i=3)$ et ceux sortants sans diplôme au-delà du bac $(i=2)$ :

$E\left(W_{33}\right)-E\left(W_{22}\right)=\left(E\left(W_{33}\right)-E\left(W_{23}\right)\right)+\left(E\left(W_{23}\right)-E\left(W_{22}\right)\right)$

Le terme (A) exprime la différence de gains issue de l'écart de caractéristiques individuelles moyennes (écart de caractéristiques entre les sortants diplômés de bac +2 et les sortants non diplômés de bac +2 ). Les coefficients d'une seule équation de salaires sont utilisés (dans l'exemple celui des diplômés de bac + 2). Ce terme peut être qualifié d'« effet des caractéristiques individuelles »).

Le terme $(B)$ traduit la différence de rémunération due au différentiel de valorisation entre les types de sortie $j=3$ et $j=2$ pour un même individu dont les caractéristiques correspondent aux individus sortis non-diplômés de bac +2 . Ce terme peut être qualifié $d$ '« effet de valorisation ".

Ce second terme peut s'interpréter comme le supplément de salaire que pourrait escompter un individu d'un niveau donné s'il était sorti de formation initiale à un niveau supérieur. 
C'est entre les jeunes en échec au niveau bac +2 et les diplômés, que les caractéristiques individuelles contribuent le plus à l'écart de salaire (126,6/190,5, le salaire plus élevé des diplômés s'expliquant donc pour les deux tiers par leurs caractéristiques spécifiques).

Concernant les bacheliers technologiques, la comparaison des gains espérés d'une poursuite non diplômante ou de l'accès à un diplôme d'enseignement supérieur montre que la poursuite d'études rapporte $10 \%$ (écart de 96,6 euros) alors que le diplôme offre une prime de $26 \%$ (cf. tableau 5). Cependant, ces valeurs surestiment l'avantage réel résultant de la poursuite d'études pour ces bacheliers puisque l'essentiel des écarts de salaire entre types de sortie relève de différences de caractéristiques entre les populations. Ainsi, si les diplômés de bac +2 gagnent en moyenne 250 euros de plus que les sortants immédiatement après l'obtention du bac, près de $88 \%$ de cet écart $(219,4$ euros) renvoie aux caractéristiques singulières des premiers relativement aux seconds (cf. tableau 5-C).

Pour les bacheliers technologiques, le rendement espéré de la poursuite d'études diplômantes relativement à une sortie dès la réussite au bac sépare de manière nette les trois populations. La prime est ainsi deux fois plus éle-

Tableau 4

Détenteur d'un bac professionnel et rendements salariaux de la poursuite d'études après le bac

A - Salaires simulés aux caractéristiques moyennes des titulaires de bac professionnel

En euros mensuels

\begin{tabular}{|l|c|c|c|}
\hline & \multicolumn{3}{|c|}{ Caractéristiques moyennes des... } \\
\hline $\begin{array}{l}\text { Rémunérations, calculées à partir des paramètres estimés des } \\
\text { équations de salaire, des caractéristiques pour une } \ldots\end{array}$ & $\begin{array}{c}\ldots \text { bac }+2 \text { diplômés } \\
(i=3)\end{array}$ & $\begin{array}{c}\ldots \text { bac }+2 \\
\text { non diplômés } \\
(i=2)\end{array}$ & $\begin{array}{c}\ldots \text { bac professionnel } \\
(i=1)\end{array}$ \\
\hline$\ldots$ sortie à bac +2 diplômés $(j=3)$ & 1229,2 & 1102,6 & 1152,7 \\
\hline$\ldots$ sortie à bac +2 , non diplômés $(j=2)$ & 1146,0 & 1038,7 & 1096,2 \\
\hline $\begin{array}{l}\ldots \text { sortie immédiatement après l'obtention du bac professionnel } \\
(j=1)\end{array}$ & 1081,9 & 1016,2 & 1020,6 \\
\hline \multicolumn{2}{|l|}{ Dans ce tableau les trois cases diagonales correspondent à des situations observées, les autres à nos évaluations. } \\
\hline
\end{tabular}

\section{B - Rendements salariaux}

\begin{tabular}{|l|c|c|c|}
\hline & $\begin{array}{c}\text { Bac }+2 \text { diplômés } \\
(i=3)\end{array}$ & $\begin{array}{c}\text { Bac }+2 \\
\text { non diplômés } \\
(i=2)\end{array}$ & $\begin{array}{c}\text { Bac professionnel } \\
(i=1)\end{array}$ \\
\hline $\begin{array}{l}\text { Rendement de la poursuite d'études diplômantes relativement } \\
\text { à une sortie immédiatement après l'obtention du bac }\end{array}$ & 13,6 & 8,5 & 12,9 \\
\hline $\begin{array}{l}\text { Rendement de l'obtention du bac + 2 relativement à une sortie } \\
\text { bac + 2 sans diplôme }\end{array}$ & 7,3 & 6,2 & \\
\hline $\begin{array}{l}\text { Rendement de la poursuite d'études sans diplôme relativement } \\
\text { à une sortie immédiatement après l'obtention du bac }\end{array}$ & & & 5,2 \\
\hline
\end{tabular}

\section{C - Décomposition des écarts de salaire prédits}

\begin{tabular}{|l|c|c|c|}
\hline \multicolumn{1}{|c|}{ Écarts de salaire prédits entre... } & Effet valorisation & $\begin{array}{c}\text { Effet des caractéris- } \\
\text { tiques individuelles }\end{array}$ & Écart total \\
\hline $\begin{array}{l}\text {... les diplômés bac }+2 \text { et les sortants immédiatement après } \\
\text { l'obtention du bac }\end{array}$ & 132,1 & 76,5 & 208,6 \\
\hline$\ldots$ les diplômés bac + 2 et les non-diplômés de bac + 2 & 63,9 & 126,6 & 190,5 \\
\hline $\begin{array}{l}\text {... les non-diplômés de bac + 2 et les sortants immédiatement } \\
\text { après l'obtention du bac }\end{array}$ & 75,6 & $-57,5$ & 18,1 \\
\hline
\end{tabular}

Lecture: dans les tableaux $A$ et $B$, si les sortants au bac professionnel (c'est-à-dire immédiatement après l'obtention du bac) étaient sortis de formation initiale diplômés de bac + 2, leur salaire aurait été en moyenne de 1 152,7 euros, soit $12,9 \%$ de plus que le salaire qu'ils perçoivent en moyenne (1 020,6 euros).

Dans le tableau C, le salaire prédit des jeunes diplômés de bac + 2 est supérieur de 208,6 euros à celui prédit pour les sortants immédiatement après leur réussite au bac (différence des valeurs diagonales du tableau A), dont 132,1 euros sont dus à l'effet valorisation - les mêmes caractéristiques sont rémunérées différemment pour chaque sortie du marché du travail -, 76,5 à des écarts de caractéristiques individuelles, (cf. encadré 3).

Source : Génération 98, 1 ère interrogation, Céreq. 
vée pour les bac +2 diplômés $(17 \%)$ qu'elle ne l'est pour les jeunes de niveau IV supérieur l'écart étant accentué par rapport aux sortants effectifs immédiatement après le bac technologique, lesquels ne peuvent espérer qu'un bonus de 3,2\%. D'autre part, pour les trois populations, les surplus salariaux escomptés de la poursuite d'études avec échec au diplôme de bac +2 sont supérieurs à ceux tirés de l'obtention du diplôme.

Contrairement aux bacheliers professionnels, les détenteurs d'un bac technologique et sortant à ce niveau auraient un faible avantage à poursuivre leurs études dans l'enseignement supérieur. En effet, du point de vue de la sélection, la situation des bacheliers technologiques quittant le système scolaire à ce niveau diffère des détenteurs d'un bac professionnel qui quittent l'école immédiatement après ce diplôme. Les premiers représentent une population très spécifique car ils se distinguent de la majorité de ces bacheliers qui continuent leurs études. À l'inverse, il continue sans doute d'exister une forte hétérogénéité de caractéristiques observées et non observées dans l'ensemble des bacheliers professionnels sortants, puisque environ $80 \%$ s'arrêtent à ce niveau. Qui plus est, les bacheliers professionnels possèdent en forte proportion des caractéristiques qui s'avèrent les mieux valorisées quand la sortie du système éducatif a lieu dès l'obtention du diplôme de niveau bac +2 (ce sont majoritairement des hommes issus de la filière industrielle notamment), le cas opposé s'observant pour les bacheliers technologiques.

Tableau 5

Détenteurs d'un bac technologique et rendements salariaux de la poursuite d'études après le bac

\section{A - Salaires simulés aux caractéristiques moyennes des titulaires de bac technologique}

\begin{tabular}{|c|c|c|c|}
\hline \multirow[b]{2}{*}{$\begin{array}{l}\text { Rémunérations, calculées à partir des paramètres estimés des } \\
\text { équations de salaire, des caractéristiques pour une... }\end{array}$} & \multicolumn{3}{|c|}{ Caractéristiques moyennes des... } \\
\hline & $\begin{array}{c}\ldots \text { bac }+2 \text { diplômés } \\
(i=3)\end{array}$ & $\begin{array}{c}\text { no bac }+2 \\
\text { non diplômés } \\
(i=2)\end{array}$ & $\begin{array}{c}\text {... bac professionnel } \\
(i=1)\end{array}$ \\
\hline ... sortie à bac + 2 diplômés $(j=3)$ & 1195,7 & 1083,9 & $976,3^{*}$ \\
\hline $\begin{array}{l}\ldots \text { sortie à bac }+2, \text { non diplômés }(j=2) \\
\ldots \text { sortie immédiatement après l'obtention du bac professionnel } \\
(j=1)\end{array}$ & $\begin{array}{l}1125,3 \\
1021,6\end{array}$ & 1042,6 & 961,4 \\
\hline
\end{tabular}

\section{B - Rendements salariaux}

\begin{tabular}{|l|c|c|c|}
\hline & $\begin{array}{c}\text { Bac + 2 diplômés } \\
(i=3)\end{array}$ & $\begin{array}{c}\text { Bac }+2 \\
\text { non-diplômés } \\
(i=2)\end{array}$ & $\begin{array}{c}\text { Bac professionnel } \\
(i=1)\end{array}$ \\
\hline $\begin{array}{l}\text { Rendement de la poursuite d'études diplômantes relativement } \\
\text { à une sortie immédiatement après l'obtention du bac }\end{array}$ & 17 & 8,6 & 3,2 \\
\hline $\begin{array}{l}\text { Rendement de l'obtention du bac + 2 relativement à une sortie } \\
\text { bac + 2 sans diplôme }\end{array}$ & 6,2 & 3,9 & 1,5 \\
\hline $\begin{array}{l}\text { Rendement de la poursuite d'études sans diplôme relativement } \\
\text { à une sortie immédiatement après l'obtention du bac }\end{array}$ & & 10 & 4,5 \\
\hline
\end{tabular}

\section{C - Décomposition des écarts de salaire prédits}

\begin{tabular}{|l|c|c|c|}
\hline \multicolumn{1}{|c|}{ Écarts de salaire prédits entre... } & Effet valorisation & $\begin{array}{c}\text { Effet des caractéris- } \\
\text { tiques individuelles }\end{array}$ & Écart total \\
\hline $\begin{array}{l}\text {... les diplômés bac + 2 et les sortants immédiatement après } \\
\text { l'obtention du bac }\end{array}$ & 30,3 & 219,4 & 249,7 \\
\hline ... les diplômés bac + 2 et les non-diplômés de bac + & 41,3 & 111,8 & 153,1 \\
\hline $\begin{array}{l}\text {... les non-diplômés de bac + 2 et les sortants immédiatement } \\
\text { après l'obtention du bac }\end{array}$ & 15,4 & 81,2 & 96,6 \\
\hline
\end{tabular}

Lecture: dans les tableaux $A$ et $B$, si les sortants immédiatement après l'obtention du bac technologique étaient sortis de formation initiale diplômés de bac +2 , alors leur salaire aurait été en moyenne de 976,3 euros, soit 3,2 \% de plus que leur salaire moyen (946 euros).

Dans le tableau C, le salaire prédit des jeunes diplômés de bac +2 est supérieur de 249,7 euros à celui prédit pour les sortants au bac (différence des valeurs diagonales du tableau A), dont 30,3 euros sont dus à des écarts de rétribution salariale - les mêmes caractéristiques sont rémunérées différemment pour chaque sortie du marché du travail - et 219,4 à des écarts de caractéristiques individuelles. Source : Génération 98, 1 ère interrogation, Céreq. 
Pour mettre en regard les rendements établis ici, spécifiques à chacune des populations et ceux obtenus de manière frustre à partir des populations totales des bacheliers professionnels et technologiques (cf. tableau 3), on calcule des rendements de la poursuite diplômante par rapport à une sortie immédiatement après l'obtention du bac, prenant en compte les effets de sélection, pour les trois sous-populations prises ensemble. Pour les titulaires d'un bac professionnel par exemple, ces rendements s'obtiennent en pondérant les rendements de 13,6\%, 8,5\% et $12,9 \%$ (cf. tableau 4-B) par les parts respectives des jeunes diplômés de bac +2 , des jeunes de niveau IV supérieur et de sortants immédiatement après l'obtention du bac parmi l'ensemble des détenteurs d'un bac professionnel. Les rendements ainsi obtenus sont de $12,4 \%$ et de $11,8 \%$ respectivement pour les bacheliers professionnels et technologiques, bien inférieurs à ceux de $21 \%$ et $18,1 \%$ (cf. tableau 3 ). La prise en compte des risques inhérents à la poursuite d'études et à l'accès à l'emploi réduit les gains attendus de la poursuite diplômante, laissant toujours l'avantage aux bacheliers professionnels sur les technologiques.

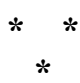

$\mathrm{Au}$ vu des rendements salariaux que peuvent attendre les bacheliers professionnels et technologiques s'ils poursuivent leurs études, tels qu'ils sont évalués ici, il semblerait que le processus d'orientation (sortie de formation initiale versus poursuite d'études post-bac) et d'auto-sélection à l'entrée dans l'enseignement supérieur s'organise de manière plutôt adéquate pour les bacheliers technologiques puisque ceux qui s'arrêtent immédiatement après l'obtention du bac n'auraient pas eu intérêt à aller plus loin. Pour les bacheliers professionnels, en revanche, l'analyse plaiderait pour une ouverture plus importante de l'enseignement supérieur aux sortants de ces filières dans la mesure où en moyenne, ils pourraient escompter un gain de la poursuite, même en cas d'échec au diplôme de niveau bac +2 .

Ces conclusions sont cependant soumises à un ensemble de limites. En particulier, le bénéfice de la poursuite d'études diplômante ou non est fondé sur un seul salaire, celui de l'emploi occupé après trois ans de vie active. Par ailleurs, la population étudiée est celle qui sort de formation initiale au niveau bac +2 ou en deçà. Elle est donc exclusive de la population des jeunes qui, par exemple, passent par un bac technologique et qui poursuivent leurs études après l'obtention du diplôme de bac +2 . Enfin, les résultats en termes de bénéfice salarial de la poursuite d'études ignorent la variabilité de ce bénéfice selon la nature de la filière de formation suivie. Des distinctions notamment entre filières universitaires et filières professionnelles de l'enseignement supérieur, et entre spécialités industrielle et tertiaire pour ces dernières, permettraient des éclairages complémentaires.

\section{BIBLIOGRAPHIE}

Balsan D. (2000), «Évaluation des rendements éducatifs dans un contexte de chômage », Économie Publique, $\mathrm{n}^{\circ}$ 5, pp. 91-119.

Blinder A. (1973), «Wage Discrimination Reduced Form and Structural Estimates », Journal of Human Resources, vol. 8, n 4, pp. 436-455.

Caille J.-P. et Vallet L.-A. (1995), « Les carrières scolaires au collège des élèves étrangers ou issus de l'immigration », Éducation et formations, $\mathrm{n}^{\circ} 40$, pp. 5-14.

Céreq (2001), Quand l'école est finie... - Premiers pas dans la vie active de la Génération 98.

Dubois M. et Raulin E. (1997), «L'entrée dans l'enseignement supérieur : permanences et changements 1982-1996 », Éducation et formations, $\mathrm{n}^{\circ} 50$, pp. 11-19.
Dupray A. et Recotillet I. (2005), « Quelle adéquation à l'emploi pour les jeunes issus d'itinéraires scolaires atypiques? », in Des formations pour quels emplois?, J.-F. Giret, A. Lopez et J. Rose éditeurs, collection Recherches, La Découverte.

Eckert H. (2002), "La place des jeunes entre mobilité et reproduction sociales ", in Quand les jeunes entrent dans l'emploi, pp. 23-40, M. Arliaud et H. Eckert éditeurs, La Dispute.

Epiphane D. et Hallier P. (1996), «Les bacheliers dans l'enseignement supérieur », Documents Observatoire, $\mathrm{n}^{\circ} 113$, Céreq, $81 \mathrm{p}$.

Fabre A. et Moullet S. (2004), « Externalités de l'éducation et mobilité intergénérationnelle : application au cas français », Économie et Prévision, $\mathrm{n}^{\circ} 166$, pp. 19-37. 
Giret J.-F., Moullet S. et Thomas G. (2003), « De l'enseignement supérieur à l'emploi : les trois premières années de vie active de la Génération 98 », Notes Emploi Formation, Céreq.

Giret J.-F., Moullet S. et Thomas G. (2003), «L'enseignement supérieur professionnalisé, un atout pour entrer dans la vie active ? », Bref, $\mathrm{n}^{\circ} 195$, Céreq.

Greene W.H. (2003), Econometric Analysis, $5^{\mathrm{e}}$ édition, Prentice Hall, New York University.

Goux D. et Maurin É. (1997), «Destinées sociales : le rôle de l'école et du milieu d'origine », Économie et Statistique, n 306, pp. 13-26.

Coëffic N. (1998), « Parcours scolaires au collège et au lycée », Note d'Information, MEN, n 98-01, janvier.

Hanchane S. et Verdier É. (2003), « $80 \%$ au bac et avant... (Questions autours de la demande d'éducation en France et de ses mécanismes) », Communication aux $\mathrm{X}^{\mathrm{e}}$ Journées d'Études sur les Données Longitudinales dans l'Analyse du marché du travail, mai.

Heckman J.J., Lochner L.J. et Todd P.E. (2003), " Fifty Years of Mincer Earnings Regressions », NBER Working Papers, $\mathrm{n}^{\circ} 9732$.

Hilmer M.J.(2001), « A Comparison of Alternative Specifications of the College Attendance Equation with an Extension to Two-Stage SelectivityCorrection Models », Economics of Education Review, vol. 20, n 3, pp. 263-278.

Lemaire S. (1998), «Que deviennent les bacheliers après leur bac ? », Note d'Information, MEN, $\mathrm{n}^{\circ} 98-05$, mars.

Lemaire S. (2004), «Que deviennent les bacheliers après leur bac? Évolutions 1996-2002», Note d'Information, MEN, $\mathrm{n}^{\circ}$ 04-14, juin.

Lévy-Garboua L. (1976), «Les demandes de l'étudiant ou les contradictions de l'université de masse », Revue Française de Sociologie, vol. XVII, $n^{\circ} 1$, pp. 53-80.
Lollivier S. et Pollet P. (2003), « Impact de la formation initiale sur les rémunérations au cours de la vie active », Revue d'Économie Politique, ${ }^{\circ} 6$, pp. 801-826.

Magnac T. et Thesmar D. (2002), "Analyse économique des politiques éducatives: l'augmentation de la scolarisation en France de 1982 à 1993 », Annales d'Économie et de Statistique, $\mathrm{n}^{\circ} 65$, pp. 1-32.

Modesto L. (2003), « Should I Stay or Should I Go? Educational Choices and Earnings: an Empirical Study for Portugal », Journal of Population Economics, vol. 16, n 2, pp. 307-322.

Moullet S. (2000), Contributions à l'analyse des rendements éducatifs : enjeux théoriques et problèmes empiriques d'identification, Thèse pour le doctorat en sciences économiques, Université de la Méditerranée, 238 p.

Oaxaca R.L. (1973), «Male-Female Wage Differentials in Urban Labor Markets », International Economic Review, vol. 14, $\mathrm{n}^{\circ} 3$, pp. 693-709.

Puhani P.A (2000), «The Heckman Correction for Sample Selection and Its Critique », Journal of Economics Surveys, vol. 14, n 1, pp. 53-68.

Terracol A. (2002), "Triprobit and the GHK Simulator: a Short Note », annexe à la commande Triprobit de Stata.

Thomas G. (2003), « Les jeunes qui sortent sans diplôme de l'enseignement supérieur », Bref, $\mathrm{n}^{\circ} 200$, Céreq.

White (1980), «A Heteroscedasticity-Consistent Covariance Matrix Estimator and a Direct Test for Heteroscedasticity », Econometrica, vol. 48, n 4 , pp. 817-838.

Willis R. et Rosen S. (1979), « Education and Self Selection », Journal of Political Economy, vol. 87, $\mathrm{n}^{\circ} 5 \mathrm{~b}, \mathrm{pp} .507-536$.

Wooldrigde J.M. (2001), Econometric Analysis of Cross Section and Panel Data, MIT Press. 


\section{CARACTÉRISTIQUES MOYENNES DES BACHELIERS «PROFESSIONNELS » ET «TECHNOLOGIQUES»}

A - Caractéristiques moyennes des bacheliers professionnels selon le type de sortie du système éducatif

\begin{tabular}{|c|c|c|c|c|}
\hline Type de sortie & $\begin{array}{l}\text { Sortants au } \\
\text { niveau du bac }\end{array}$ & Poursuivants & $\begin{array}{c}\text { Bac }+2 \\
\text { non diplômés }\end{array}$ & $\begin{array}{l}\text { Bac }+2 \\
\text { diplômés }\end{array}$ \\
\hline $\begin{array}{l}\text { Homme } \\
\text { Retard en sixième } \\
\text { Absence d'emploi pendant les études } \\
\text { Existence d'emploi de vacances }\end{array}$ & $\begin{array}{l}59,7 \\
31,9 \\
68,5 \\
56,3\end{array}$ & $\begin{array}{l}49,3 \\
32,3 \\
72,5 \\
64,7\end{array}$ & $\begin{array}{l}46,6 \\
34,4 \\
74,7 \\
64,5\end{array}$ & $\begin{array}{l}54,6 \\
27,9 \\
68,1 \\
65,3\end{array}$ \\
\hline $\begin{array}{l}\text { Type de classe de troisième } \\
\text { Troisième générale } \\
\text { Autres troisièmes }\end{array}$ & $\begin{array}{l}77,5 \\
22,5\end{array}$ & $\begin{array}{l}83,1 \\
16,9\end{array}$ & $\begin{array}{l}80,4 \\
19,6\end{array}$ & $\begin{array}{l}88,4 \\
11,6\end{array}$ \\
\hline $\begin{array}{l}\text { Classe suivie après la troisième } \\
\text { Seconde générale } \\
\text { Seconde technologique } \\
\text { BEP - CAP apprentissage } \\
\text { BEP - CAP voie scolaire } \\
\text { (Total BEP - CAP) } \\
\text { Autres secondes }\end{array}$ & $\begin{array}{r}10,6 \\
5,8 \\
10,4 \\
68,5 \\
(78,9) \\
4,6\end{array}$ & $\begin{array}{r}15,1 \\
7,4 \\
3,7 \\
70,2 \\
(73,9) \\
3,6\end{array}$ & $\begin{array}{r}13,9 \\
5,6 \\
3,7 \\
72,6 \\
(76,3) \\
4,1\end{array}$ & $\begin{array}{r}17,5 \\
10,8 \\
3,7 \\
65,5 \\
(69,2) \\
2,6\end{array}$ \\
\hline $\begin{array}{l}\text { Spécialité du bac } \\
\text { Industrielle } \\
\text { Tertiaire } \\
\text { Agricole }\end{array}$ & $\begin{array}{l}51,2 \\
48,8\end{array}$ & $\begin{array}{r}27,2 \\
68,4 \\
4,4\end{array}$ & $\begin{array}{r}24,1 \\
72,0 \\
3,9\end{array}$ & $\begin{array}{r}33,5 \\
61,0 \\
5,5\end{array}$ \\
\hline $\begin{array}{l}1^{\text {ère }} \text { année immédiatement après le bac } \\
1^{\text {ère }} \text { année dans le } 1^{\text {er }} \text { cycle universitaire } \\
1^{\text {ère }} \text { année en STS } \\
\text { Autres }\end{array}$ & & & $\begin{array}{r}38,4 \\
55,4 \\
6,2\end{array}$ & $\begin{array}{r}3,1 \\
83,9 \\
13,0\end{array}$ \\
\hline $\begin{array}{l}\text { Type de sortie au niveau bac + } 2 \\
\text { Diplômé de BTS industriel } \\
\text { BTS tertiaire } \\
\text { Échec en Deug } \\
\text { Échec en filière pro. industrielle } \\
\text { Échec en filière pro. Tertiaire } \\
\text { (Échec à l'issue d'une filière pro.) }\end{array}$ & & & $\begin{array}{c}42,7 \\
23,4 \\
33,9 \\
(57,3)\end{array}$ & $\begin{array}{l}38,3 \\
61,7\end{array}$ \\
\hline $\begin{array}{l}\text { Catégorie sociale de la mère à la fin des études } \\
\text { Artisan, commerçant } \\
\text { Cadre } \\
\text { Technicien } \\
\text { Employé } \\
\text { Ouvrier } \\
\text { Inactive, chômeur }\end{array}$ & $\begin{array}{r}8,9 \\
4,4 \\
3,4 \\
51,1 \\
12,3 \\
19,7\end{array}$ & $\begin{array}{r}7,0 \\
5,2 \\
6,6 \\
53,7 \\
7,6 \\
20,0\end{array}$ & $\begin{array}{r}7,3 \\
5,5 \\
6,7 \\
51,1 \\
6,9 \\
22,5\end{array}$ & $\begin{array}{r}6,3 \\
4,5 \\
6,4 \\
59,0 \\
8,9 \\
14,8\end{array}$ \\
\hline $\begin{array}{l}\text { Catégorie sociale du père à la fin des études } \\
\text { Artisan, commerçant } \\
\text { Cadre } \\
\text { Technicien } \\
\text { Employé } \\
\text { Ouvrier } \\
\text { Inactif, chômeur }\end{array}$ & $\begin{array}{r}17,5 \\
7,5 \\
8,7 \\
29,5 \\
27,6 \\
9,3\end{array}$ & $\begin{array}{r}15,0 \\
11,5 \\
9,2 \\
30,6 \\
23,2 \\
10,5\end{array}$ & $\begin{array}{r}14,0 \\
9,8 \\
7,5 \\
30,6 \\
25,3 \\
12,8\end{array}$ & $\begin{array}{r}17,2 \\
14,8 \\
12,6 \\
30,6 \\
19,0 \\
5,8\end{array}$ \\
\hline $\begin{array}{l}\text { Père français ou né en France } \\
\text { Mère française ou née en France }\end{array}$ & $\begin{array}{l}88,0 \\
89,0\end{array}$ & $\begin{array}{l}80,1 \\
82,1\end{array}$ & $\begin{array}{l}74,3 \\
76,8\end{array}$ & $\begin{array}{l}91,6 \\
92,6\end{array}$ \\
\hline
\end{tabular}

Lecture: parmi les jeunes détenteurs d'un bac professionnel et sortis du système éducatif immédiatement après l'obtention de ce diplôme (en 1998), 59,70\% sont des hommes. Parmi les jeunes qui obtiennent un diplôme de bac +2 après un bac professionnel, $54,6^{\circ} \%$ sont des hommes.

Source : Génération 98, 1 ère interrogation, Céreq.

B - Caractéristiques moyennes des bacheliers technologiques selon le type de sortie du système éducatif

\begin{tabular}{|l|c|c|c|c|}
\multicolumn{1}{|c|}{ Type de sortie } & $\begin{array}{c}\text { Sortants au } \\
\text { niveau du bac }\end{array}$ & Poursuivants & $\begin{array}{c}\text { Bac }+2 \\
\text { non diplômés }\end{array}$ & $\begin{array}{c}\text { Bac }+2 \\
\text { diplômés }\end{array}$ \\
\hline Homme & 37,3 & 55,4 & 49,8 & 60,1 \\
Retard en sixième & 19,6 & 15,5 & 18,8 & 12,8 \\
Absence d'emploi pendant les études & 87,1 & 84,6 & 82,7 & 86,1 \\
Existence d'emploi de vacances & 61,3 & 73,0 & 72,7 & 73,3 \\
\hline Type de classe de troisième & & & & \\
Troisième générale & 91,3 & 95,2 & 94,6 & 95,7 \\
Autres troisièmes & 8,7 & 4,8 & 5,4 & 4,3 \\
\hline
\end{tabular}




\begin{tabular}{|c|c|c|c|c|}
\hline $\begin{array}{l}\text { Classe suivie après la troisième } \\
\text { Seconde générale } \\
\text { Seconde technologique } \\
\text { BEP - CAP apprentissage } \\
\text { BEP - CAP voie scolaire } \\
\text { (Total BEP - CAP) } \\
\text { Autres secondes }\end{array}$ & $\begin{array}{r}42,5 \\
18,8 \\
3,2 \\
34,5 \\
(37,7) \\
1,0\end{array}$ & $\begin{array}{r}55,2 \\
27,9 \\
1,0 \\
15,5 \\
(16,5) \\
0,5\end{array}$ & $\begin{array}{c}56,4 \\
23,1 \\
1,1 \\
18,9 \\
(20,0) \\
0,5\end{array}$ & $\begin{array}{r}54,1 \\
31,9 \\
0,8 \\
12,7 \\
(13,5) \\
0,5\end{array}$ \\
\hline $\begin{array}{l}\text { Spécialité du bac } \\
\text { Industrielle } \\
\text { Tertiaire }\end{array}$ & $\begin{array}{l}26,8 \\
73,2\end{array}$ & $\begin{array}{l}38,7 \\
61,3\end{array}$ & $\begin{array}{l}30,0 \\
70,0\end{array}$ & $\begin{array}{l}46,0 \\
54,0\end{array}$ \\
\hline $\begin{array}{l}1^{\text {ère }} \text { année immédiatement après le bac } \\
1^{\text {ère }} \text { année dans le } 1^{\text {er }} \text { cycle universitaire } \\
1^{\text {ère }} \text { année en STS } \\
1^{\text {ère }} \text { année en IUT } \\
\text { Autres }\end{array}$ & & & $\begin{array}{r}34,1 \\
51,1 \\
9,0 \\
5,7\end{array}$ & $\begin{array}{r}6,5 \\
75,5 \\
14,5 \\
3,5\end{array}$ \\
\hline $\begin{array}{l}\text { Type de sortie au niveau bac + } \mathbf{2} \\
\text { Diplômé de BTS industriel } \\
\text { BTS tertiaire } \\
\text { DUT industriel } \\
\text { DUT tertiaire } \\
\text { Deug } \\
\text { Échec en Deug } \\
\text { Échec en filière pro. industrielle } \\
\text { Échec en filière pro. tertiaire } \\
\text { (Échec à l'issue d'une filière pro.) }\end{array}$ & & & $\begin{array}{c}42,3 \\
22,0 \\
35,7 \\
(57,7)\end{array}$ & $\begin{array}{r}37,7 \\
45,8 \\
6,2 \\
8,0 \\
2,3\end{array}$ \\
\hline $\begin{array}{l}\text { Catégorie sociale de la mère à la fin des études } \\
\text { Artisan, commerçant } \\
\text { Cadre } \\
\text { Technicien } \\
\text { Employé } \\
\text { Ouvrier } \\
\text { Inactive, chômeur }\end{array}$ & $\begin{array}{r}8,1 \\
5,8 \\
5,1 \\
53,7 \\
12,5 \\
14,8 \\
\end{array}$ & $\begin{array}{r}6,8 \\
7,8 \\
4,7 \\
52,7 \\
9,6 \\
18,4 \\
\end{array}$ & $\begin{array}{r}5,6 \\
6,1 \\
4,2 \\
53,0 \\
10,0 \\
21,0 \\
\end{array}$ & $\begin{array}{r}7,7 \\
9,2 \\
5,1 \\
52,4 \\
9,3 \\
16,2 \\
\end{array}$ \\
\hline $\begin{array}{l}\text { Catégorie sociale du père à la fin des études } \\
\text { Artisan, commerçant } \\
\text { Cadre } \\
\text { Technicien } \\
\text { Employé } \\
\text { Ouvrier } \\
\text { Inactif, chômeur }\end{array}$ & $\begin{array}{r}16,2 \\
10,5 \\
11,4 \\
29,4 \\
23,4 \\
9,1\end{array}$ & $\begin{array}{r}15,6 \\
14,8 \\
11,0 \\
28,0 \\
21,2 \\
9,3\end{array}$ & $\begin{array}{r}14,1 \\
13,5 \\
8,8 \\
28,5 \\
23,7 \\
11,4\end{array}$ & $\begin{array}{r}16,9 \\
15,9 \\
12,8 \\
27,6 \\
19,2 \\
7,6\end{array}$ \\
\hline $\begin{array}{l}\text { Père français ou né en France } \\
\text { Mère française ou née en France }\end{array}$ & $\begin{array}{l}90,0 \\
91,1\end{array}$ & $\begin{array}{l}85,1 \\
86,3\end{array}$ & $\begin{array}{l}80,0 \\
81,9\end{array}$ & $\begin{array}{l}89,5 \\
90,1\end{array}$ \\
\hline
\end{tabular}

Lecture: parmi les jeunes détenteurs d'un bac technologique et sortis du système éducatif immédiatement après l'obtention de ce diplôme (en 1998), 37,3% sont des hommes. Parmi les jeunes qui obtiennent un diplôme de bac +2 après un bac technologique, $60,1^{\circ} \%$ sont des hommes.

Source : Génération 98, 1 ère interrogation, Céreq.

\section{C - Moyennes des probabilités, introduites dans les régressions de gains}

\begin{tabular}{|l|c|c|c|c|c|c|}
\hline & \multicolumn{3}{|c|}{ Bac professionnels } & \multicolumn{3}{c|}{ Bac technologiques } \\
\cline { 2 - 7 } & Sortants & $\begin{array}{c}\text { Sortants } \\
\text { non diplômés de } \\
\text { niveau bac + 2 }\end{array}$ & $\begin{array}{c}\text { Sortants } \\
\text { diplômés de } \\
\text { niveau bac + }\end{array}$ & Sortants & $\begin{array}{c}\text { Sortants } \\
\text { non diplômés de } \\
\text { niveau bac + }\end{array}$ & $\begin{array}{c}\text { Sortants } \\
\text { diplômés de } \\
\text { niveau bac + } 2\end{array}$ \\
\hline $\begin{array}{l}\text { Probabilité d'em- } \\
\text { ploi }\end{array}$ & $0,894^{*}$ & 0,861 & 0,937 & 0,77 & 0,823 & 0,923 \\
\hline $\begin{array}{l}\text { Probabilité d'échec } \\
\text { au diplôme }\end{array}$ & $0,936^{* *}$ & 0,93 & 0,912 & 0,60 & 0,561 & 0,524 \\
\hline $\begin{array}{l}\text { Probabilité de sor- } \\
\text { tie après le bac }\end{array}$ & $0,807^{\star * *}$ & 0,729 & 0,753 & 0,242 & 0,176 & 0,161 \\
\hline
\end{tabular}

Lecture: (*) en moyenne, les bacheliers professionnels sortis du système éducatif immédiatement après ce diplôme ont $89,4^{\circ} \%$ de chances d'occuper un emploi après trois ans de vie active. Lorsque les bacheliers professionnels poursuivent leurs études au-delà et quittent l'enseignement supérieur avec un diplôme de bac +2 , leur probabilité moyenne d'être en emploi trois ans après la fin des études est de $93,7^{\circ} \%$.

${ }^{* *}$ Sur la base des caractéristiques individuelles des bacheliers professionnels sortant à ce niveau, caractéristiques prises en compte dans les régressions, une probabilité d'échec au diplôme de niveau bac +2 estimée de 93,6 \% peut leur être associée. Cette probabilité est très supérieure à celle qui l'on associe aux sortants au bac technologique (60\%).

$\left.{ }^{* * *}\right)$ Compte tenu de leurs caractéristiques prises en compte dans les régressions, la probabilité estimée associée aux sortants immédiatement après l'obtention du bac professionnel est de 80,7\%, supérieure à la probabilité estimée associée aux sortants diplômés de niveau bac $+2(75,3 \%)$.

Source : Génération 98, 1 ère interrogation, Céreq. 
D - Caractéristiques moyennes d'emploi des bacheliers professionnels selon le type de sortie du système éducatif

\begin{tabular}{|l|c|c|c|}
\hline \multicolumn{1}{|c|}{ Type de sortie } & $\begin{array}{c}\text { Sortants au } \\
\text { niveau du bac }\end{array}$ & $\begin{array}{c}\text { Bac + 2 } \\
\text { non diplômés }\end{array}$ & $\begin{array}{c}\text { Bac }+2 \\
\text { diplômés }\end{array}$ \\
\hline Catégorie sociale en 2001 & 3,6 & 2,4 & 1,1 \\
Artisan, commerçant & 20,9 & 34 & 56,2 \\
Cadre et profession intermédiaire & 30,6 & 42 & 33,4 \\
Employé & 44,9 & 21,6 & 9,3 \\
Ouvrier & & & \\
\hline Type de contrat de travail & 68,8 & 63,3 & 77,3 \\
Cdi ou fonctionnaire & 13,5 & 15,4 & 15,6 \\
Cdd & 10,0 & 15,6 & 4,5 \\
Contrats aidés & 7,7 & 5,7 & 2,6 \\
Autres contrats & 85,4 & 83,7 & 93,9 \\
\hline Travail à temps plein & & & \\
\hline
\end{tabular}

Lecture: parmi les jeunes détenteurs d'un bac professionnel et sortis du système éducatif immédiatement après l'obtention de ce diplôme (en 1998) et en emploi en $2001,20,9^{\circ}$ \% sont en emploi de cadre ou profession intermédiaire. Parmi les jeunes qui obtiennent un diplôme de bac +2 après un bac professionnel, $56,2^{\circ} \%$ sont dans ce cas.

Champ : jeunes en emploi en mars 2001.

Source : Génération 98, 1 ère interrogation, Céreq

E - Caractéristiques moyennes d'emploi des bacheliers technologiques selon le type de sortie du système éducatif

\begin{tabular}{|l|c|c|c|}
\hline \multicolumn{1}{|c|}{ Type de sortie } & $\begin{array}{c}\text { Sortants au } \\
\text { niveau du bac }\end{array}$ & $\begin{array}{c}\text { Bac +2 } \\
\text { non diplômés }\end{array}$ & $\begin{array}{c}\text { Bac +2 } \\
\text { diplômés }\end{array}$ \\
\hline Catégorie sociale en 2001 & 3 & 1,5 & 1,4 \\
Artisan, commerçant & 27,3 & 39,4 & 59 \\
Cadre et profession intermédiaire & 44,2 & 38,7 & 27,7 \\
Employé & 25,5 & 20,4 & 11,9 \\
Ouvrier & & & \\
\hline Type de contrat de travail & 51,3 & 57,4 & 14,3 \\
Cdi ou fonctionnaire & 22 & 15,6 & 6,9 \\
Cdd & 18,3 & 18,2 & 5,6 \\
Contrats aidés & 8,4 & 8,8 & 94 \\
Autres contrats & 82,7 & 86,9 & \\
\hline Travail à temps plein & & & \\
\hline
\end{tabular}

En \%

Lecture : parmi les jeunes détenteurs d'un bac technologique et sortis du système éducatif immédiatement après l'obtention de ce diplôme (en 1998) et en emploi en 2001, 27,3% sont en emploi de cadre ou profession intermédiaire. Parmi les jeunes qui obtiennent un diplôme de bac +2 après un bac technologique, $59^{\circ} \%$ sont dans ce cas.

Champ : jeunes en emploi en mars 2001.

Source : Génération 98, 1 ère interrogation, Céreq. 
ANNEXE 2

\section{LES ESTIMATIONS DES ÉQUATIONS DE SALAIRE POUR LES DIFFÉRENTS GROUPES DE SORTANTS DE FORMATION INITIALE}

\section{A - Le cas des bacheliers professionnels}

\begin{tabular}{|c|c|c|c|c|c|c|}
\hline & \multicolumn{2}{|c|}{ Sortants au niveau du bac } & \multicolumn{2}{|c|}{ Non-diplômés de bac +2} & \multicolumn{2}{|c|}{ Diplômés de bac +2} \\
\hline & Coefficient & $P$-value & Coefficient & $P$-value & Coefficient & $P$-value \\
\hline Constante & 6,1521 & 0,000 & 5,4569 & 0,000 & 6,2706 & 0,000 \\
\hline Homme & $0,1093^{*}$ & 0,000 & 0,0911 & 0,001 & $0,1214^{\star}$ & 0,002 \\
\hline Temps plein & 0,2616 & 0,000 & 0,3253 & 0,000 & 0,3532 & 0,000 \\
\hline Âge en 1998 & 0,0112 & 0,000 & 0,0052 & 0,413 & $-0,0051$ & 0,627 \\
\hline Ancienneté & 0,0053 & 0,000 & 0,0039 & 0,001 & 0,0099 & 0,000 \\
\hline Expérience & 0,0054 & 0,000 & 0,0061 & 0,000 & 0,0102 & 0,000 \\
\hline Spécialité industrielle du bac & 0,0674 & 0,000 & 0,1368 & 0,000 & 0,1661 & 0,001 \\
\hline \multirow{3}{*}{$\begin{array}{l}\text { Probabilité estimée d'emploi } \\
\text { Probabilité estimée d'échec } \\
\text { au diplôme } \\
\text { Probabilité estimée de sortie } \\
\text { après le bac }\end{array}$} & 0,4553 & $0,001^{\star *}$ & 0,8862 & 0,003 & 1,0349 & 0,243 \\
\hline & $-0,1041$ & 0,423 & 0,3136 & 0,282 & $-0,1516$ & 0,709 \\
\hline & $-0,3071$ & 0,000 & $-0,2351$ & 0,090 & $-0,8076$ & 0,002 \\
\hline $\begin{array}{l}\mathrm{R}^{2} \\
\mathrm{~N}\end{array}$ & \multicolumn{2}{|c|}{$\begin{array}{c}0,2622 \\
3553\end{array}$} & \multicolumn{2}{|c|}{$\begin{array}{c}0,3529 \\
594\end{array}$} & \multicolumn{2}{|c|}{$\begin{array}{c}0,2629 \\
328\end{array}$} \\
\hline
\end{tabular}

Lecture : $\left(^{*}\right)$ toutes choses égales par ailleurs, être un homme assure un supplément de salaire de $10,9 \%$ au sein de la population des bacheliers professionnels qui entrent sur le marché du travail immédiatement après ce diplôme. Ce gain additionnel est de 12,1 \% pour les bacheliers professionnels qui sont diplômés de bac +2 .

$\left.{ }^{* *}\right)$ Les caractéristiques individuelles déterminant la probabilité d'emploi, résumées au travers la probabilité estimée ont un effet positif significatif au seuil de $1 \%$ sur la détermination des salaires pour les bacheliers professionnels sortant à ce niveau.

\section{B - Le cas des bacheliers technologiques}

\begin{tabular}{|c|c|c|c|c|c|c|}
\hline & \multicolumn{2}{|c|}{ Sortants au niveau du bac } & \multicolumn{2}{|c|}{ Non-diplômés de bac +2} & \multicolumn{2}{|c|}{ Diplômés de bac +2} \\
\hline & Coefficient & $P$-value & Coefficient & $P$-value & Coefficient & $P$-value \\
\hline Constante & 6,2163 & 0,000 & 5,9541 & 0,000 & 5,7631 & 0,000 \\
\hline Homme & $0,1596^{*}$ & 0,000 & 0,0933 & 0,000 & $0,1349^{\star}$ & 0,000 \\
\hline Temps plein & 0,3184 & 0,000 & 0,3229 & 0,000 & 0,3708 & 0,000 \\
\hline Âge en 1998 & 0,0114 & 0,096 & 0,0114 & 0,001 & 0,0104 & 0,005 \\
\hline Ancienneté & 0,0057 & 0,000 & 0,0053 & 0,000 & 0,0076 & 0,000 \\
\hline Expérience & 0,0069 & 0,000 & 0,0059 & 0,000 & 0,0090 & 0,000 \\
\hline Spécialité industrielle du bac & 0,0052 & 0,819 & 0,0761 & 0,000 & 0,0326 & 0,006 \\
\hline \multirow{3}{*}{$\begin{array}{l}\text { Probabilité estimée d'emploi } \\
\text { Probabilité estimée d'échec } \\
\text { au diplôme } \\
\text { Probabilité estimée de sortie } \\
\text { après le bac }\end{array}$} & $-0,1016$ & $0,605^{\star \star}$ & 0,3405 & 0,046 & 0,5297 & 0,045 \\
\hline & $-0,0164$ & 0,891 & $-0,0298$ & 0,709 & $-0,0337$ & 0,636 \\
\hline & 0,0080 & 0,944 & $-0,1311$ & 0,139 & $-0,2667$ & 0,001 \\
\hline $\begin{array}{l}\mathrm{R}^{2} \\
\mathrm{~N}\end{array}$ & \multicolumn{2}{|c|}{$\begin{array}{c}0,3009 \\
1039\end{array}$} & \multicolumn{2}{|c|}{$\begin{array}{c}0,2684 \\
2337\end{array}$} & \multicolumn{2}{|c|}{$\begin{array}{c}0,2578 \\
3104\end{array}$} \\
\hline
\end{tabular}

Lecture : (*) toutes choses égales par ailleurs, être un homme assure un supplément de salaire de 15,9\% au sein de la population des bacheliers technologiques qui entrent sur le marché du travail immédiatement après ce diplôme. Ce gain additionnel est de 13,5\% pour les bacheliers technologiques qui sont diplômés de bac +2 .

(*) Les caractéristiques individuelles déterminant la probabilité d'emploi, résumées au travers la probabilité estimée n'ont pas d'effet significatif sur la détermination des salaires pour les bacheliers technologiques sortant à ce niveau. 